\title{
HortNet417v1-A Deep-Learning Architecture for the Automatic Detection of Pot-Cultivated Peach Plant Water Stress
}

\author{
Md Parvez Islam ${ }^{1}$ (D) and Takayoshi Yamane ${ }^{2, *}$ \\ 1 Research Center for Agricultural Robotics, NARO, Tsukuba 3050856, Japan; p.islam@affrc.go.jp \\ 2 Research Center for Agricultural Information Technology and National Institute of Fruit Tree and Tea Science, \\ NARO, Tsukuba 3050856, Japan \\ * Correspondence: yamanet@affrc.go.jp; Tel.: +81-29-838-6502
}

Citation: Islam, M.P.; Yamane, T.

HortNet417v1-A Deep-Learning

Architecture for the Automatic

Detection of Pot-Cultivated Peach

Plant Water Stress. Sensors 2021, 21,

7924. https://doi.org/10.3390/

s21237924

Academic Editor: Junliang Xing

Received: 4 November 2021

Accepted: 25 November 2021

Published: 27 November 2021

Publisher's Note: MDPI stays neutral with regard to jurisdictional claims in published maps and institutional affiliations.

Copyright: (c) 2021 by the authors. Licensee MDPI, Basel, Switzerland. This article is an open access article distributed under the terms and conditions of the Creative Commons Attribution (CC BY) license (https:/ / creativecommons.org/licenses/by/ $4.0 /)$.

\begin{abstract}
The biggest challenge in the classification of plant water stress conditions is the similar appearance of different stress conditions. We introduce HortNet417v1 with 417 layers for rapid recognition, classification, and visualization of plant stress conditions, such as no stress, low stress, middle stress, high stress, and very high stress, in real time with higher accuracy and a lower computing condition. We evaluated the classification performance by training more than 50,632 augmented images and found that HortNet417v1 has $90.77 \%$ training, $90.52 \%$ cross validation, and $93.00 \%$ test accuracy without any overfitting issue, while other networks like Xception, ShuffleNet, and MobileNetv2 have an overfitting issue, although they achieved 100\% training accuracy. This research will motivate and encourage the further use of deep learning techniques to automatically detect and classify plant stress conditions and provide farmers with the necessary information to manage irrigation practices in a timely manner.
\end{abstract}

Keywords: deep learning; network architecture; stem water potential; plant water stress; classification

\section{Introduction}

Water management is significant for controlling fruit tree growth, yield, and fruit quality [1]. Irrigation methods for fruit trees are often determined by soil drying; however, the condition of part of the soil does not always match that of the whole soil in the root zone, because fruit tree roots are distributed at various depths depending on the soil type and floor management [2]. Plant-based irrigation is a significantly better method than soil-based estimation to save water, maintain optimal plant growth, and improve fruit quality [3]. Stem water potential is a widely accepted method to measure plant water status $[4,5]$. However, these measurements are destructive and labor intensive. Therefore, a low-cost and non-destructive method is needed.

Due to the tremendous development and reduction in cost of image acquisition, data storage, and computing systems, the computer vision technique has become a popular tool for deep learning (DL). DL in general is the successor of machine learning with many hidden layers and massive training data [6] with the capability of an automatic acquiring for hierarchical feature and learning via non-linear filters from the bottom and thereafter producing decision-making or classification at the top [7]. Deep learning emphasizes the depth of the deep network structure to learn complex features with a higher level of accuracy, enabling a network to solve problem-specific tasks such as object detection, semantic segmentation, and image analysis. It has been reported that many well-known DL architectures, such as LeNet, AlexNet, VGG, ResNet, GoogleNet, MobileNet, Inception, and SqueezeNet, are widely used to identify and classify leaf diseases [8]. In agriculture, it is difficult to separate the object from the background because of their similarity. On the other hand, the dynamic nature of the background in orchards and farmland creates challenges for the classification task. 
Wakamori et al. [9] proposed a multimodal neural network with long short-term memory layers. Their network requires physiological and meteorological data to estimate plant water stress with a $21 \%$ mean absolute and root-mean-squared error. Their target plant was a rockwool-cultivated tomato grown in a greenhouse with a very short and shallow rootzone. For physiological data, they used images of leaf wilting. The leaf-wilting phenomenon is mostly related to daytime transpiration [10]. This might be helpful in plants that grow inside a greenhouse. However, leaf wilt also depends on leaf age and weather conditions, such as rainfall and wind. Our target plant is the peach tree, which is long and deep rooted and grown mostly in open-field conditions. Soil-moisture conditions mostly depend on the soil type, and both moisture and soil type are non-uniform in depth and distance from the plant. The water uptake capacity also varies from plant to plant, and transpiration varies among different parts of the peach tree.

Kamarudin et al. [11] conducted a comprehensive review of various plant water-stress assessment methods and reported that most of the existing deep-learning solutions are based on soil moisture estimation, soil water modelling, evapotranspiration estimation, evapotranspiration forecasting, plant water-stress estimation, and plant water-stress identification. All of these methods use sensory measurement data for machine learning analysis, which mostly depends on the quality of the data and the specific target application [12]. However, deep learning is mostly used in disease detection and yield prediction.

The author proposed a new DL architecture with several components (convolutions, batch normalization, ReLU, max pooling, depth concatenation, element wise addition, dropout, fully connected layers, softmax, classification output layer, etc.). In some literature $[13,14]$, it is explained that deepening the DL network could increase classification accuracy with a noisy background. Therefore, the depth of the DL architecture is fixed to 417 layers and accurately optimized based on training performance. The aim of this work is to demonstrate the HortNet417v1 (horticulture network 417 version 1) performance for the automatic detection of peach plant leaf water stress under various environmental conditions.

\section{Materials and Methods}

Nine 9-year-old peach trees (Prunus persica (L.) Batsch cv. 'Akatsuki' grafted onto 'Ohatsumomo' peach), were planted in $60 \mathrm{~L}$ plastic pots in a greenhouse at the National Institute of Fruit Tree and Tea Science, National Agriculture and Food Research Organization (NARO) in Tsukuba, Ibaraki, Japan. This experiment was conducted from 27 May to 19 June 2020. Full bloom was 28 March and the growth stage of these trees was from 65 (stage II of the fruit-developing stage) to 88 days (stage III of that two weeks before harvest) after full bloom. The cultivation process is shown in Figure 1.

The pot cultivation method was used to simulate the water stress condition. The stem water potential method verified the stress conditions of each peach tree. Three irrigation methods were applied to create different stress conditions: (1) Wet treatment: when the soil water potential reached $-0.005 \mathrm{MPa}$, three potted trees were automatically irrigated to maintain moist conditions. (2) Dry treatment: when the soil water potential reached $-0.050 \mathrm{MPa}$, three potted trees were automatically irrigated to maintain that level. But during the experiment, the irrigation was stopped four times until the soil water potential reached about $-0.070 \mathrm{MPa}$.

A tensiometer (DM-8HG, Takemura Denki Seisakusho Co., Ltd., Tokyo, Japan) was used to monitor the soil water potential at a depth of $15 \mathrm{~cm}$ every $10 \mathrm{~min}$ and photographs were taken of these tress under various stress conditions and different water conditions. Two matured leaves per tree were enclosed in a small hermetic aluminum foil bag at least $30 \mathrm{~min}$ before the measurement time. These leaves reached equilibrium with the main stem of the tree; therefore, their water potential represented the whole tree. This method is known to be stable to estimate the water condition of the whole tree [15,16]. The stem water potential of each tree was measured 22 times under various stress conditions from 27 May to 19 June 2020. A pressure chamber (Model 600, PMS Instrument Company, Albany, OR, 
USA) was used for this experiment. Photographs were taken using a smart phone equipped with a camera (iPhone 11, Apple, Cupertino, CA, USA) (Figure 2).

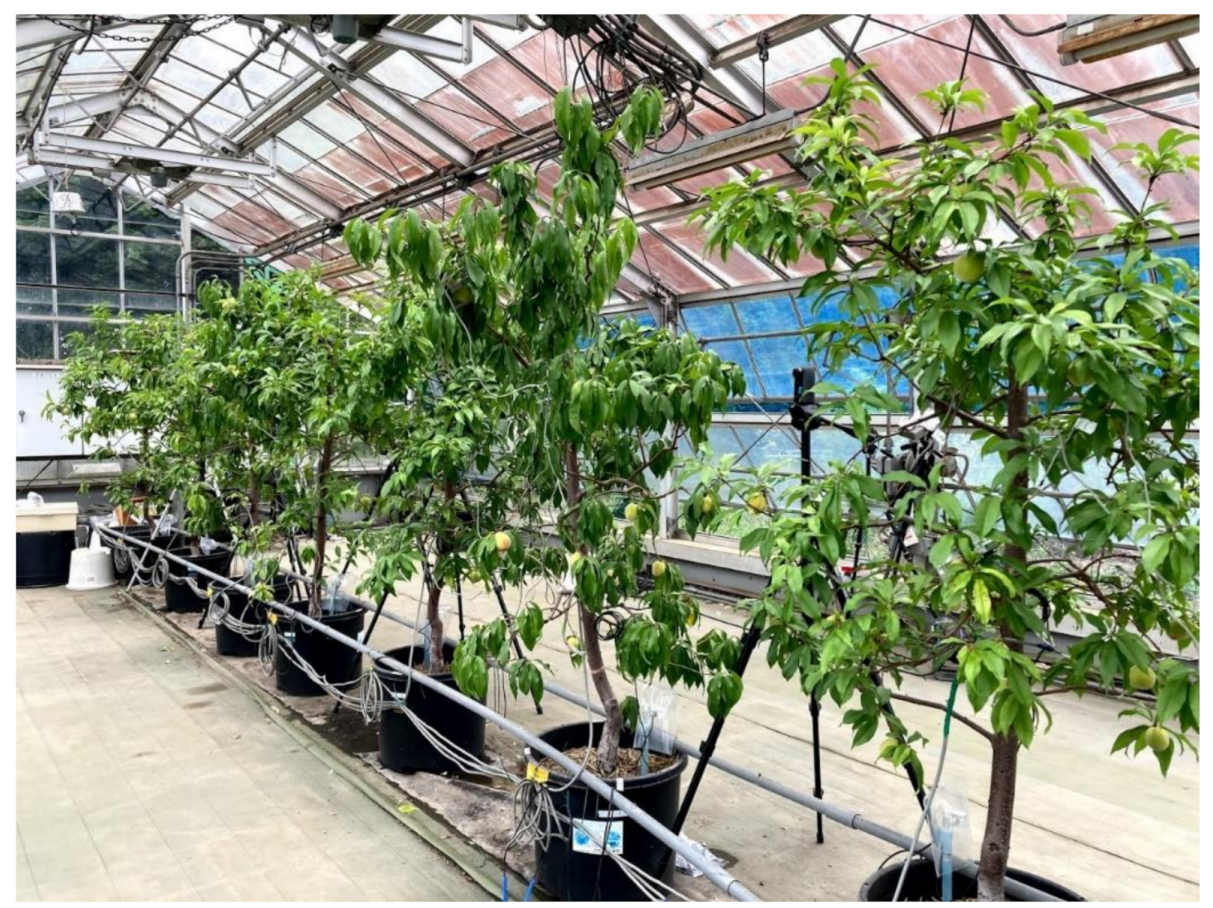

Figure 1. The planted peach tree cultivation system.

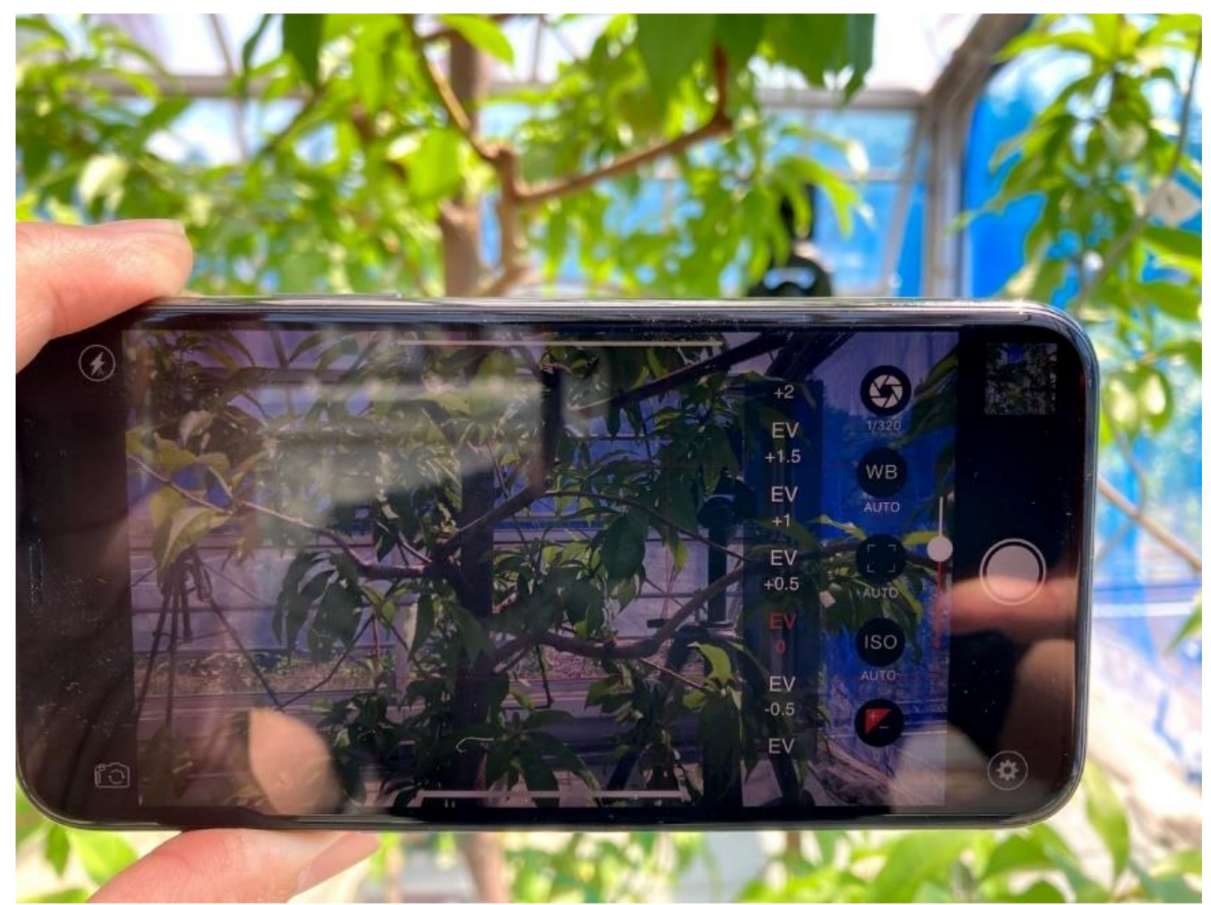

Figure 2. Image-acquisition process.

We randomly take around 100 image per tree to ensure the data diversity under various illumination conditions (shaded/sunlit), air temperatures, proximate distances, and points of view (direction and height). A total of 25,000 images were obtained during the experiment. Water-stress conditions were divided into five classes for subsequent analysis by stem water potential: (1) no stress, over $-0.5 \mathrm{MPa}$; (2) low stress, -0.5 to 
$-1.0 \mathrm{MPa}$; (3) moderate stress, -1.0 to $-1.5 \mathrm{MPa}$; (4) high stress, -1.5 to $-2.0 \mathrm{MPa}$; (5) very high stress, under $-2.0 \mathrm{MPa}$.

The proposed network architecture of HortNet417v1 represents a semantic segmentationbased network (convolution layers) and a classification-based network (softmax), as shown in Figure 3.

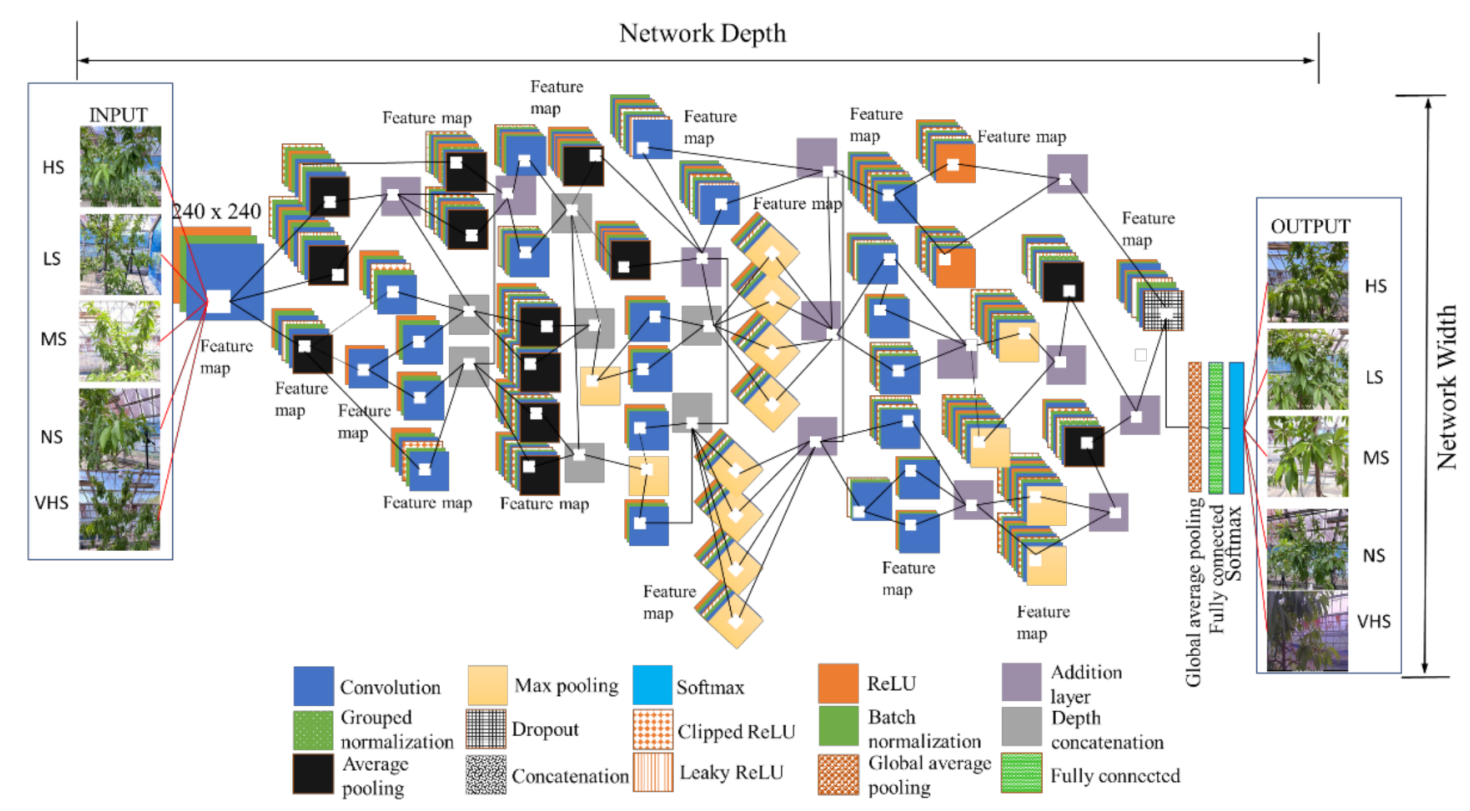

Figure 3. HortNet417v1 network architecture (HS—high stress, LS-low stress, MS—moderate stress, NS—no stress, VHS—very high stress).

The higher-level image features extracted from input image have multiple and smaller filter sizes of $3 \times 3 \times 3 \times 32$ pixels in the second layer of the network. The convolution in the second layer has a strong generalization ability that improves the network initial learning performance even when the input images are conglutinated with various objects [17].

The ReLU (rectified linear unit) activation function adds non-linearities to the proposed network, and converts the value of each input element that is less than zero to zero by performing a threshold operation [18]. The ReLU layer also converts the total weighted input from the node to the output, which overcomes the vanishing gradient problem. This also enables the model to learn faster and perform with higher accuracy. However, ReLU is not continuously differentiable and sometimes leads to cause the dying ReLU problem (neuron death). To prevent this, a LeakyReLU is added, where any input value less than zero is multiplied by a fixed scalar (fixed manually). This will not saturate with the positive value of the input, so it can prevent the gradient from exploding/disappear during the gradient descent process.

A ClippedReLU reduces the feature value of the output from becoming too large [19]. The batch-normalization layer transforms each input in the mini-batch, subtracts the batch mean, and then divides the mini-batch by the standard deviation [20]. This layer normalized the output from a previous activation layer and increased the network stability.

In addition, in order to avoid the use of mini-batch dimensions, we introduce a group normalization layer, which divides the input channels into groups and normalizes across each group [21]. The max pooling layer simplifies the complexity of the network by compressing features for fast computing, and extracting main features, thereby ensuring the invariance of feature positions and rotations [17,22]. The average pooling layer creates a downsampled (pooled) feature by dividing the input into rectangular regions, computing the average values of each region, adding a small amount of translation invariance, and extracting smooth features, whereas max pooling mostly extracts features like edges [23,24]. 
The dropout in the 404th layer randomly drops $10 \%$ of neurons in order to prevent the overfitting issue. The last convolution of the 411th layer has 64 filters, with batch normalization and ReLU activation, and then global average pooling. We added global average pooling to the 414 th layer $(1 \times 1 \times 64)$ before the fully connected layer. This layer downsamples the height and width dimensions of the input, and reduces the total number of parameters without sacrificing performance and minimizes overfitting. After multiple rounds of convolution and pooling, all abstract features are integrated into a vector through a fully connected layer. This layer has five outputs, corresponding to five classes that feed into the softmax layer for calculating the probability of the output classification layer. These expanded features passed to the classification layer for classification [25]. Several performance metrics, such as training/validation/test accuracy, confusion matrices, and validation loss and training time, evaluate the proposed deep-learning network architecture. The confusion matrices are given in terms of percentage and absolute number. Therefore, the depth of the DL architecture is fixed to 417 layers and accurately optimized based on training performance. The statistics of the HortNet417v1 architecture are shown in Table 1.

Table 1. Statistics of the HortNet417v1 architecture.

\begin{tabular}{lclc}
\hline \multicolumn{1}{c}{ Layer Name } & $\begin{array}{c}\text { Total Number } \\
\text { of Layers }\end{array}$ & \multicolumn{1}{c}{ Layer Name } & $\begin{array}{c}\text { Total Number } \\
\text { of Layers }\end{array}$ \\
\hline Image Input & 1 & Group Normalization & 6 \\
Convolution & 124 & Addition layer & 12 \\
ReLU & 65 & Depth Concatenation & 7 \\
Clipped ReLU & 31 & Global Average Pooling & 1 \\
Leaky ReLU & 25 & Concatenation & 1 \\
Dropout (10\%) & 1 & Fully Connected & 1 \\
Batch Normalization & 112 & Softmax & 1 \\
Average Pooling & 14 & Pixel classification (Output) & 1 \\
Max Pooling & 14 & & \\
\hline
\end{tabular}

The learning parameters applied to train HortNet417v1 were validation frequency: 50; validation patience: inf; mini-batch size: 260; maximum epoch: 50; learn rate schedule: piecewise; shuffle: every epoch; initial learn rate: 0.001 ; learn rate drop period: 10; learn rate drop factor: 0.1; L2 regularization: 0.0001; sequence length: longest; sequence padding value: 0 ; sequence padding direction: right; and epsilon: $1.00 \mathrm{E}-08$. The adaptive moment estimation (ADAM) was used to optimize the network weights. All analyses were run using the Supercomputer FUJITSU SHIHO equipped with TESLA V100 -SXM2 32GB and CUDA version 10.2, Deep Learning and Parallel computing Toolbox (Matlab R2020a).

For comparative analysis, we used modified pretrained network NasNet-Mobile, ResNet-50, Xception, ShuffleNet, SqueezeNet, GoogleNet, and MobileNetv2, as shown in Table 2.

Table 2. Network for comparative analysis.

\begin{tabular}{cccc}
\hline Network & $\begin{array}{c}\text { Number of } \\
\text { Layers }\end{array}$ & Input Image Size & References \\
\hline NasNet-Mobile & 913 & $224 \times 224 \times 3$ & {$[26]$} \\
ResNet-50 & 177 & $224 \times 224 \times 3$ & {$[27]$} \\
Xception & 170 & $299 \times 299 \times 3$ & {$[28]$} \\
ShuffleNet & 172 & $224 \times 224 \times 3$ & {$[29]$} \\
SqueezeNet & 68 & $227 \times 227 \times 3$ & {$[30]$} \\
GoogleNet & 144 & $224 \times 224 \times 3$ & {$[31]$} \\
MobileNetv2 & 154 & $224 \times 224 \times 3$ & {$[32]$} \\
HortNet417v1 & 417 & $240 \times 240 \times 3$ & - \\
\hline
\end{tabular}

We designed HortNet417v1 in such a way that allows it to be used in both lowpowered mobile and high-powered fixed devices. Several performance metrics, such as 
training accuracy (TA), validation accuracy (VA), test accuracy (TeA), confusion matrix, training loss (TL), validation loss (VL), sensitivity (Equation (1)), specificity (Equation (2)), precision (Equation (3)), F1 score (Equation (4), and Matthews correlation coefficient (MCC, Equation (5)) are used to evaluate HortNet417v1 network efficiency. However, $\Delta$ Accuracy $_{T r-V a l}$ in Equation (6) and $\Delta \operatorname{Loss}_{T r-V a l}$ in Equation (7) are used to evaluate HortNet417v1 network overfitting issue. The same performance metrics are also evaluated on NasNet-Mobile, ResNet-50, Xception, ShuffleNet, SqueezeNet, GoogleNet, and MobileNetv2 for comparative analysis. Visualization of the predicted stress condition for evaluating the accuracy based on validation (6266 images) and test (500 images) data are also provided in the Results Section 3 and Discussion Section 4.

$$
\text { Sensitivity }=\frac{\mathrm{TP}}{\mathrm{TP}+\mathrm{FN}}
$$

where, $\mathrm{TP}$ - true positive, $\mathrm{FN}$-false negative, worst value $=0$, best value $=+1$.

$$
\text { Specificity }=\frac{\mathrm{TN}}{\mathrm{TN}+\mathrm{FP}}
$$

where, $\mathrm{TN}$ - true negative, worst value $=0$, best value $=+1$.

$$
\text { Precision }=\frac{\mathrm{TP}}{\mathrm{TP}+\mathrm{FP}}
$$

where, worst value $=0$, best value $=+1$.

$$
\mathrm{F} 1-\text { score }=2 \times \frac{(\text { Sensitivity } \times \text { Precision })}{(\text { Sensitivity }+ \text { Precision })}
$$

where, worst value $=0$, best value $=+1$.

$$
\mathrm{MCC}=\frac{\operatorname{Cov}(\mathrm{c}, 1)}{\sigma_{\mathrm{c}} \times \sigma_{1}}=\frac{\mathrm{TP} \times \mathrm{TN}-\mathrm{FP} \times \mathrm{FN}}{\sqrt{(\mathrm{TP}+\mathrm{FP}) \times(\mathrm{TP}+\mathrm{FN}) \times(\mathrm{TN}+\mathrm{FP}) \times(\mathrm{TN}+\mathrm{FN})}}
$$

where, $\operatorname{Cov}(c, l)$ is the covariance of the true classes $c$ and predicted labels $l, \sigma_{c}$ and $\sigma_{1}$ are the standard deviations, worst value $=-1$, best value $=+1$.

$$
\begin{gathered}
\Delta \text { Accuracy }_{\text {Tr }-\mathrm{Val}}=\frac{\mathrm{TA}-\mathrm{VA}}{100} \times 100 \\
\Delta \operatorname{Loss}_{\mathrm{Tr}-\mathrm{Val}}=\frac{\mathrm{TL}-\mathrm{VL}}{100} \times 100
\end{gathered}
$$

We employed a network visualization feature using the deep dream function and compute the network layer activation to visualize the activation of different layers of the network. This also demonstrates how networks identify and learn features in different depths of the network. The t-SNE (t-distributed stochastic neighbor embedding) algorithm is used to reduce the high dimensionality and visualize the low-dimension data in a way that respects the similarities between data points [33]. We use occlusion sensitivity and locally interpretable model-agnostic explanation (LIME) techniques to predict the result evaluation (what/why/how/which/where). The occlusion sensitivity technique demonstrates what input image features are used by the network to make a classification decision and helps to identify the reason behind the network misclassification decision [34]. The LIME technique generates synthetic data from the input, classifying the synthetic data using the net and then using a simpler, more interpretable machine-learning classification/regression model to fit the classification results [35].

Figure 4 shows the pattern of image datasets for the training, validation, and testing of HortNet $417 \mathrm{v} 1$. In this experiment, $70 \%$ of the randomly selected images from the image dataset were used for training and 30\% for validation purposes. An independent 500-image 
dataset (100 images/stress condition) was used for testing purposes. The image dataset was augmented, including random $X$ and $Y$ reflection, to prevent overfitting and to generalize the model for better network learning. A total of 43,866 augmented images were used to train the HortNet417v1 network.
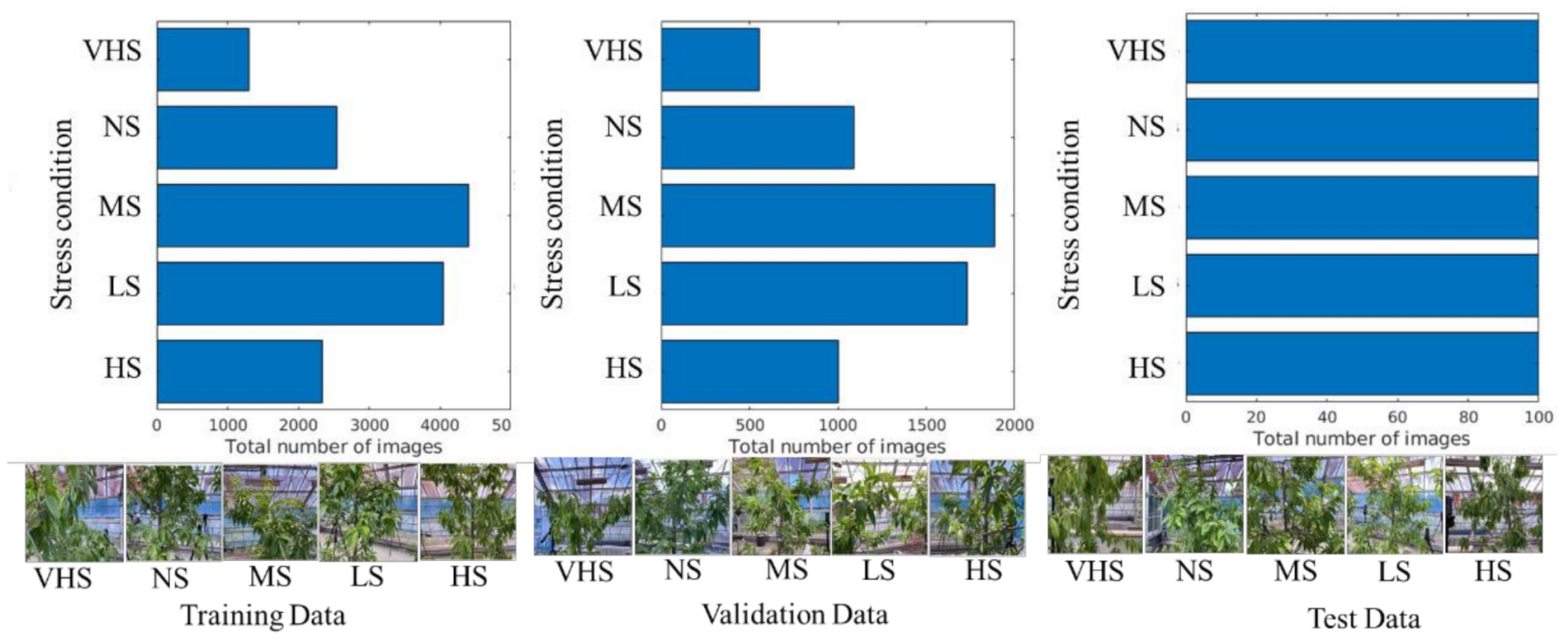

Figure 4. Visualization of the input image dataset.

Figure 5 visualizes the experimental step (from dataset preparation to networks training, model evaluation and prediction) for network analysis.

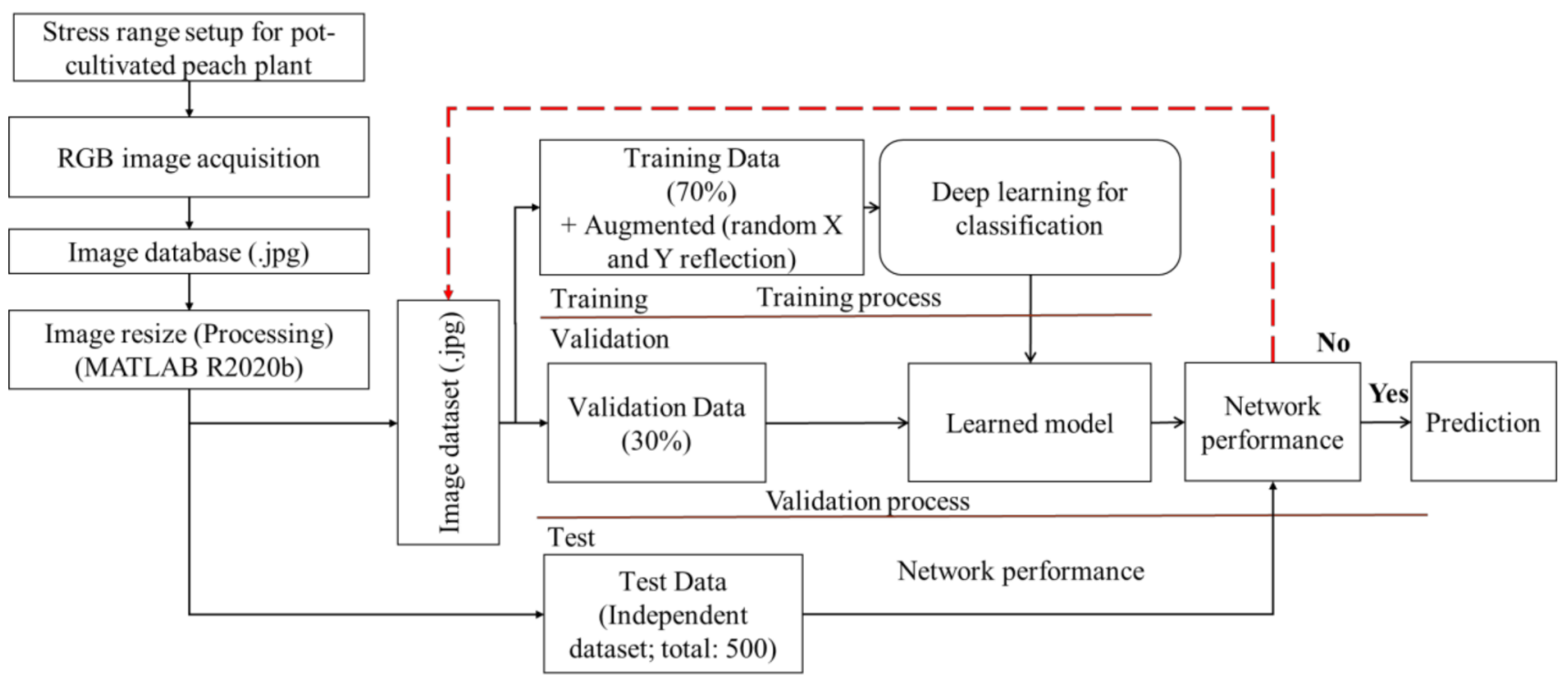

Figure 5. Schematic diagram of the network image dataset preparation and analysis.

\section{Results}

\subsection{Performance Evaluation}

Table 3 shows the result of several performance metrics for evaluating HortNet417v1 network efficiency. This comparative analysis shows that HortNet417v1 can classify stress conditions with the test accuracy as high as NasNet-Mobile/Resnet-50/Xception/ShuffleNet/ MobileNetv2 and higher than GoogleNet or SqueezeNet. From Table 3, we can see that Xception, ShuffleNet, and MobileNetv2 reached the convergence stage after 29.35, 29.23, and 19.3 min of network training, then stopped at 25, 24, and 26 max. epochs, respectively, with $100 \%$ training accuracy for all three networks, lower validation accuracy of $96.18 \%$, 
$92.60 \%$ and $94.13 \%$ and slightly higher test accuracy of $97.20 \%, 93.60 \%$ and $95.40 \%$. This trend demonstrated that models with higher training accuracy can be overfit by memorizing properties of the training set but failed to predict with validation or test data with same accuracy level [36]. The class imbalance problem of the training dataset might also affect the lower training loss $(2 \%, 3 \%$ and $2 \%)$ and higher validation loss $(11 \%, 21 \%, 20 \%)$ for Xception, ShuffleNet, and MobileNetv2, respectively. However, this class imbalance problem did not affect our proposed model HortNet417v1 network performance which shows very close training and validation loss of $21 \%$ and $20 \%$, respectively.

Table 3. Training performance (comparative analysis).

\begin{tabular}{lccccccc}
\hline Network & $\begin{array}{c}\text { Time } \\
\text { (Min) }\end{array}$ & $\begin{array}{c}\text { Max } \\
\text { Epoch }\end{array}$ & TA (\%) & VA (\%) & TeA (\%) & TL (\%) & VL (\%) \\
\hline NasNet- & 225.58 & 25 & 98.50 & 96.10 & 96.80 & 3.00 & 11.00 \\
Mobile & 26.35 & 25 & 98.85 & 94.56 & 94.00 & 4.00 & 16.00 \\
ResNet-50 & 29.23 & 24 & 100 & 96.18 & 97.20 & 2.00 & 11.00 \\
Xception & 23.18 & 22 & 100 & 92.60 & 93.60 & 3.00 & 21.00 \\
ShuffleNet & 6.54 & 27 & 58.85 & 59.16 & 62.00 & 89.00 & 87.00 \\
SqueezeNet & 1.31 & 3 & 28.85 & 30.08 & 20.00 & 15.50 & 15.20 \\
GoogleNet & 19.3 & 26 & 100 & 94.13 & 95.40 & 2.00 & 20.00 \\
MobileNetv2 & 213 & 36 & 90.77 & 90.52 & 93.00 & 21.00 & 20.00 \\
HortNet417v1 & 213 & & & & & &
\end{tabular}

NasNet-Mobile and ResNet-50 reached their convergence stage after 225.58 and $26.35 \mathrm{~min}$ at 25 max epochs with $98.50 \%$ and $98.85 \%$ training accuracy, respectively. SqueezeNet and GoogleNet achieved their convergence stage after 6.54 and 1.31 min of network training, then stopped at 27 and 3 max epochs with only $58.85 \%$ and $28.85 \%$ training accuracy, respectively. Our proposed HortNet417v1 network reached its convergence stage after 213 min of training, then stopped at 36 max epochs with 90.77\% training accuracy.

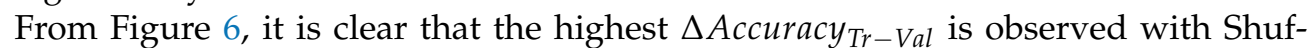
fleNet (7.4\%), MobileNetv2 (5.87\%), ResNet-50 (4.29\%), NasNet-Mobile (2.4\%), and Xception $(3.82 \%)$, while a negative $\Delta A c c u r a c y_{T r}-V a l$ is found with GoggleNet $(-1.23 \%)$ and SqueezeNet $(-0.31 \%)$. The lowest $\Delta$ Accuracy $_{T r-V a l}$ is observed with the HortNet417v1 $(0.25 \%)$ network, and this demonstrated that the network learns without any overfitting issue. On the other hand, the highest $\Delta \operatorname{Loss}_{T r-V a l}$ is observed with GoogleNet $(0.03 \%)$ and SqueezeNet $(0.02 \%)$, and the negative $\Delta$ Loss $_{T r-V a l}$ is observed with MobileNetv2 $(-0.18 \%)$, ShuffleNet $(-0.18 \%)$, ResNet-50 $(-0.12 \%)$, Xception $(-0.09 \%)$, and NasNetMobile $(-0.08 \%)$. The lowest $\Delta \operatorname{Loss}_{T r-V a l}$ is also observed with the HortNet417v1 $(0.01 \%)$ network. In both cases ( $\Delta$ differences between training and validation accuracy and $\Delta$ differences between training and validation loss), the network demonstrates stability, and it is possible to improve network performance by adding more data.

It can be seen from the confusion chart in Figure 7a, with a validation dataset, that the higher classification accuracy of $96.5 \%$ was achieved for the very high stress condition, followed by no stress (92.6\%), moderate stress $(87.1 \%)$, low stress $(89.7 \%)$ and high stress $(93.2 \%)$.

However, the confusion chart in Figure $7 \mathrm{~b}$, with a test dataset, also demonstrated that this accuracy increased from $92.6 \%$ to $98.9 \%$ (very high stress), from $92.6 \%$ to $96.9 \%$ (no stress), and from $87.1 \%$ to $90.3 \%$ (moderate stress); meanwhile, it fell slightly from $89.7 \%$ to $89.0 \%$ (low stress) and from $93.2 \%$ to $90.5 \%$ (high stress). This performance can be improved by adding more data patterns with a training dataset.

Figure 8 shows the performance indicators of each single class. Even when the data of each class is unbalanced, the HortNet417v1 network achieves the best value for all individual classes. 


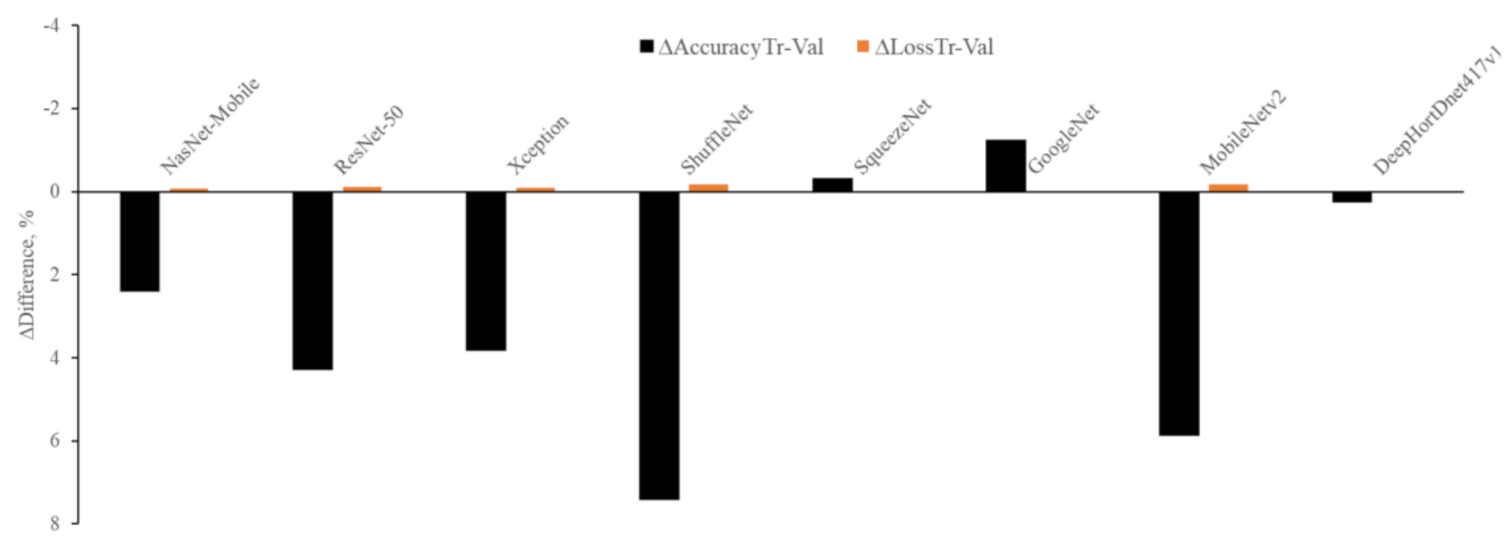

Figure 6. Visualization of network stability.

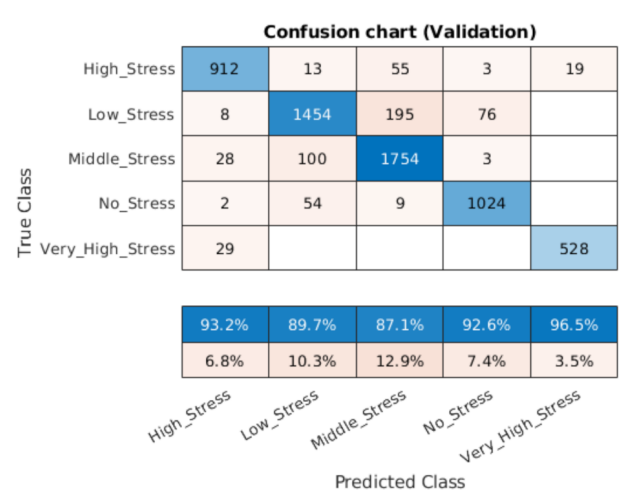

(a)

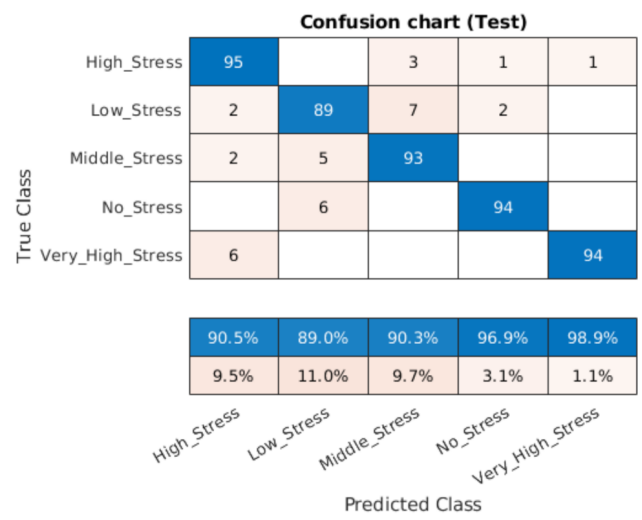

(b)

Figure 7. Confusion matrix with validation (a) and test (b) datasets.

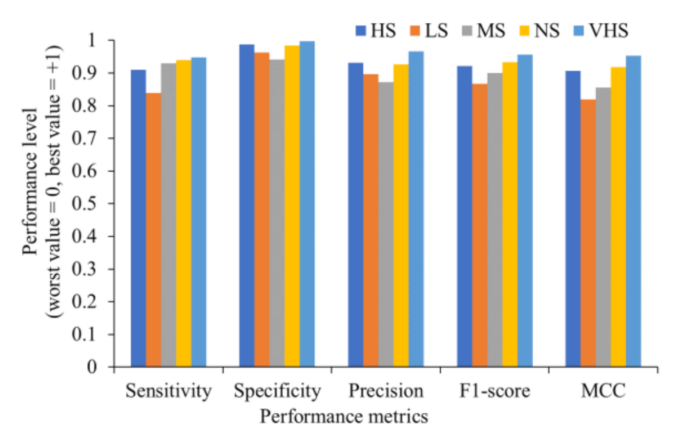

(a) Validation data

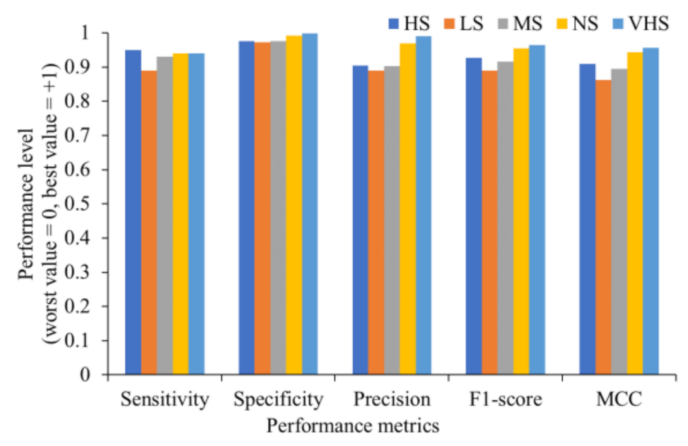

(b) Test data

Figure 8. Evaluation of the individual stress condition.

\subsection{Visualization of the Predicted Stress Condition for Evaluating Accuracy Based on Test Data}

Figure 9a shows 20 randomly selected predicted images to evaluate the accuracy based on 6266 validation and 500 image data sets. The network is perfectly classified based on the validation and test images, and in both cases, as shown in Figure 9a,b, only one misclassification occurred. This issue was caused by the similar plant responses under low and moderate stress conditions (low stress: -0.5 to $-1.0 \mathrm{MPa}$; moderate stress: -1.0 to $-1.5 \mathrm{MPa}$ ). By adding various patterns with the training dataset for low and moderate stress conditions, especially in a range from $-0.8 \mathrm{MPa}$ to $-1.2 \mathrm{MPa}$, it is possible to overcome this problem. 

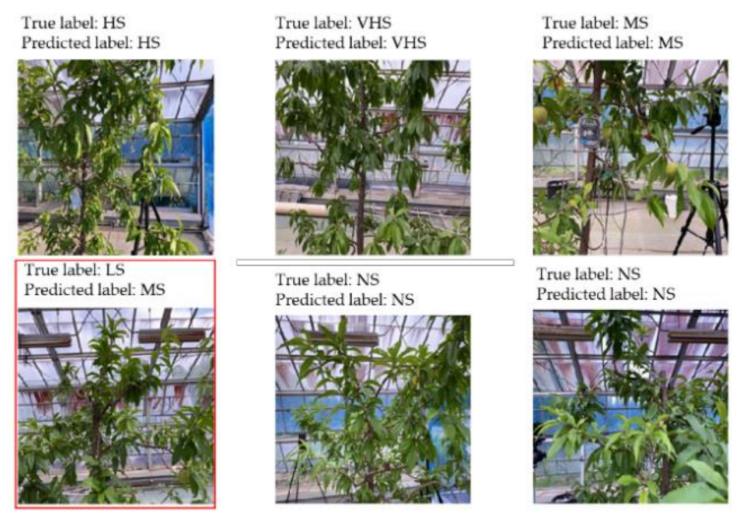

True label: MS

Predicted label: NS

True label: NS
Predicted label: NS
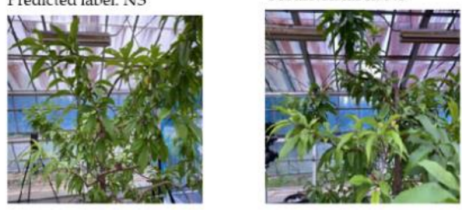

Predicted label: MS

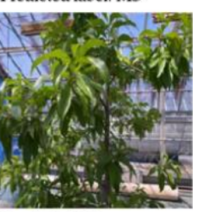

True label: LS

Predicted label: LS

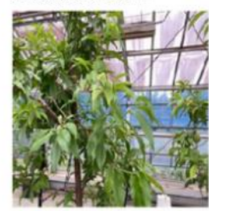

True label: VHS

Predicted label: VHS

True label: LS

Predicted label: LS

(a)

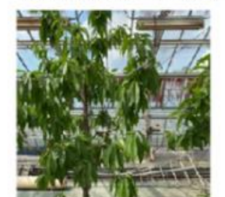

True label: HS

Predicted label: HS

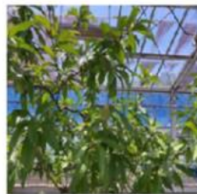

True label: VHS

Predicted label: VHS

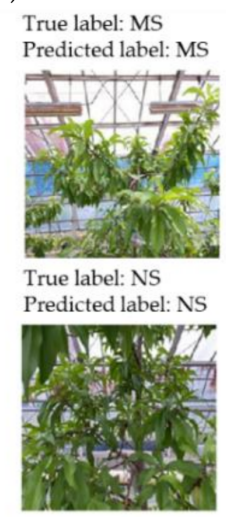

True label: LS

Predicted label: LS
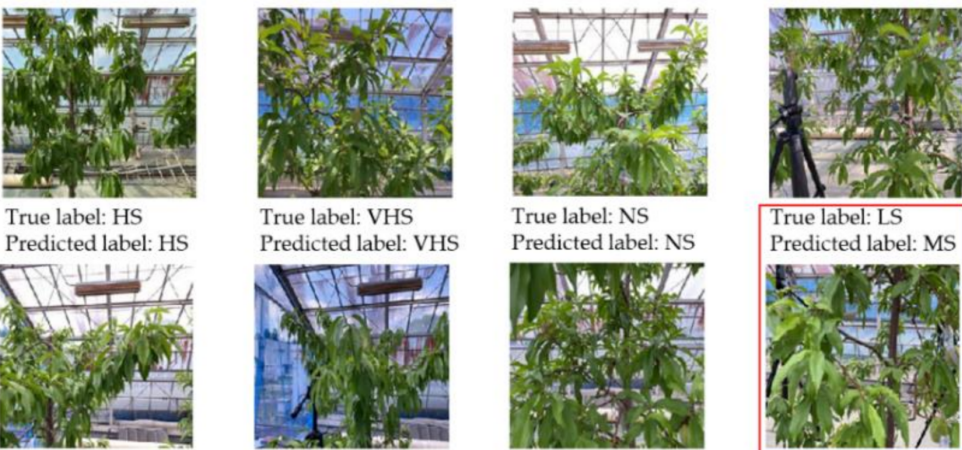

True label: LS

Predicted label: MS

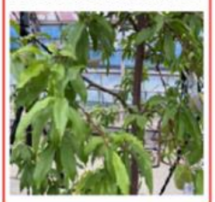

(b)

Figure 9. Prediction based on validation (a) and test (b) image datasets.

\subsection{Visualization of the Network Feature and Layer Activations}

Figure 10 Visualization of the network feature using deep dream (i, iii, v, vii) and layer activations (ii, iv, vi, viii) at different depths of the HortNet417v1 network.

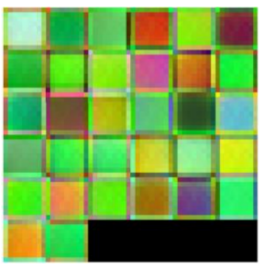

(i)

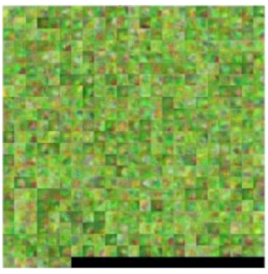

(v)

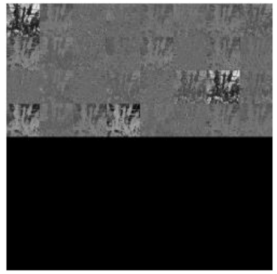

(ii)

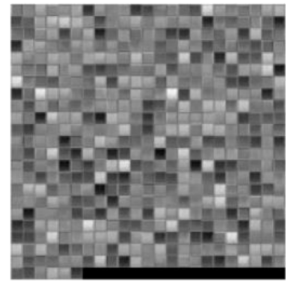

(vi)

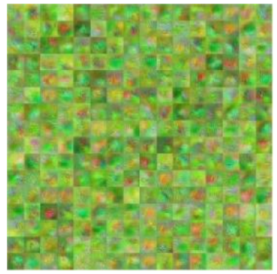

(iii)

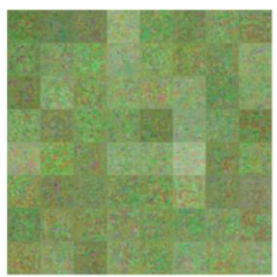

(vii)

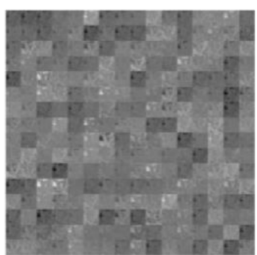

(iv)

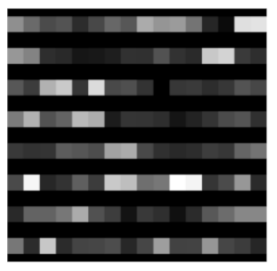

(viii)

(a) Convolution of different depths in the network layer

Figure 10. Cont. 


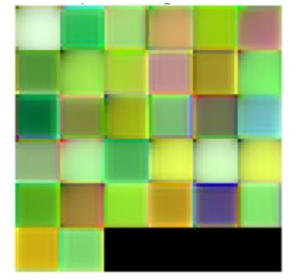

(i)

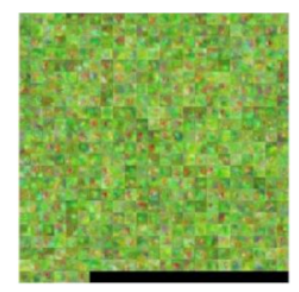

(v)

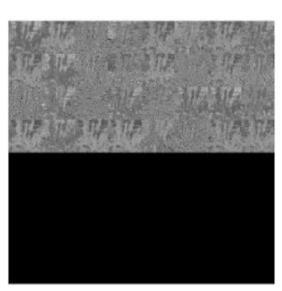

(ii)

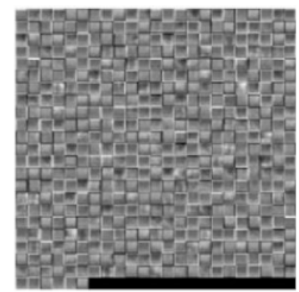

(vi)

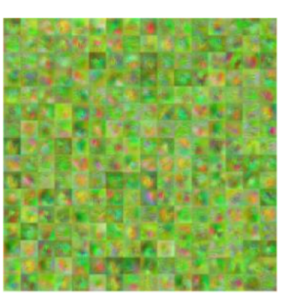

(iii)

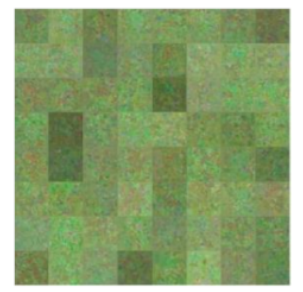

(vii)

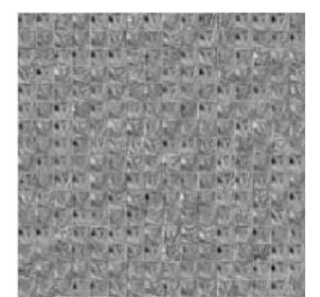

(iv)

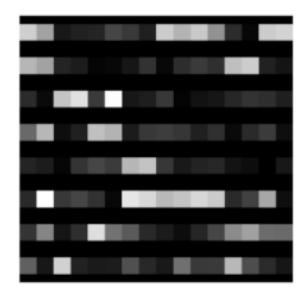

(viii)

(b) Batch normalization of different depths in the network layer.

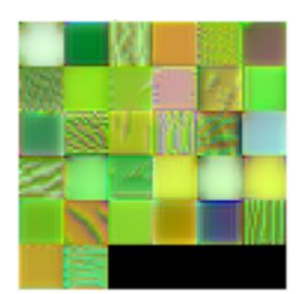

(i)

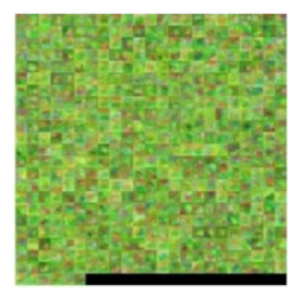

(v)

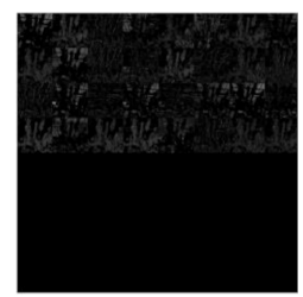

(ii)

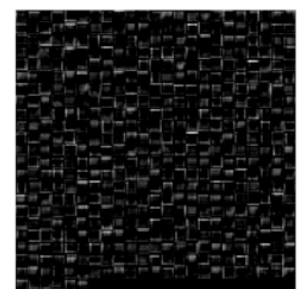

(vi)

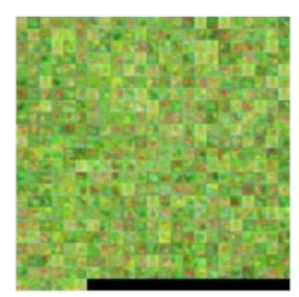

(iii)

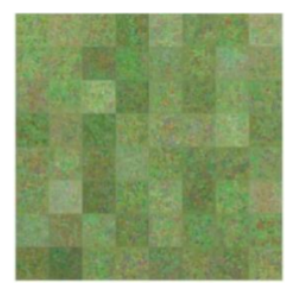

(vii)

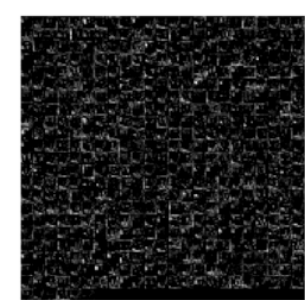

(iv)

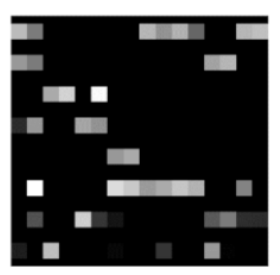

(viii)

(c) Rectified linear unit (ReLU) of different depths in the network layer

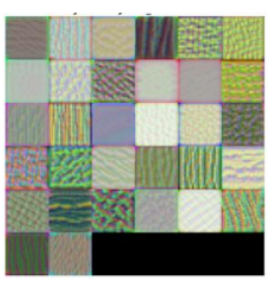

(i)

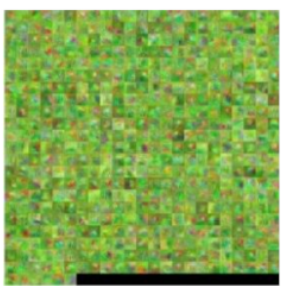

(v)

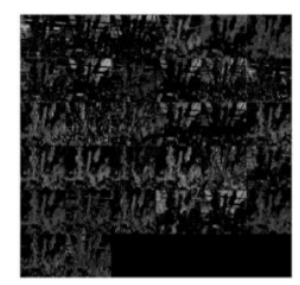

(ii)

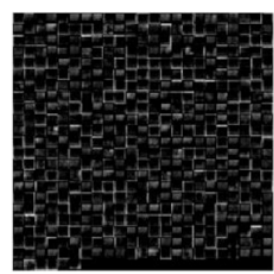

(vi)

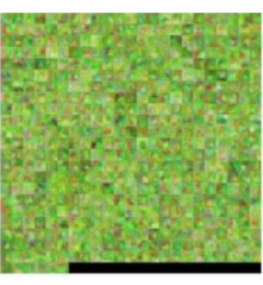

(iii)

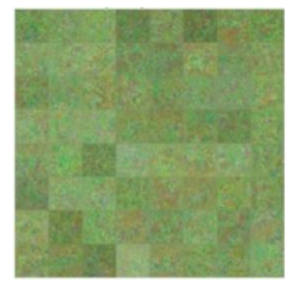

(vii)

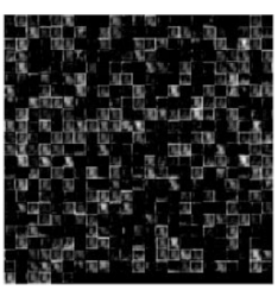

(iv)

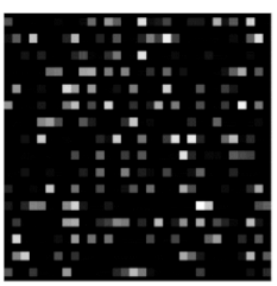

(viii)

(d) LeakyReLU of different depths in the network layer

Figure 10. Cont. 


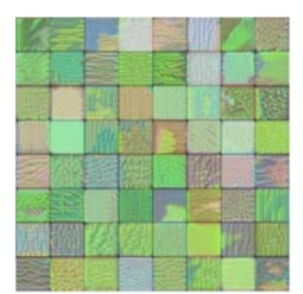

(i)

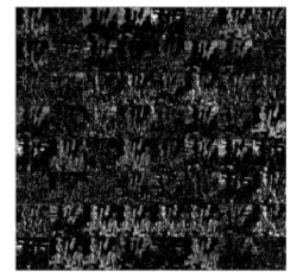

(ii)

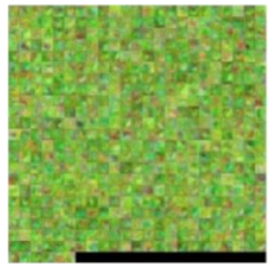

(iii)

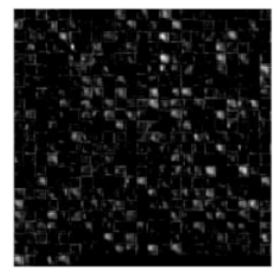

(iv)

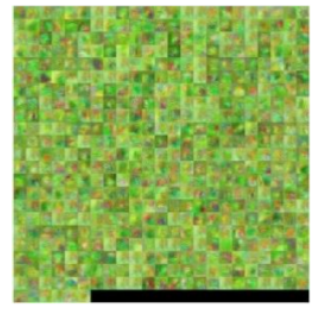

(v)

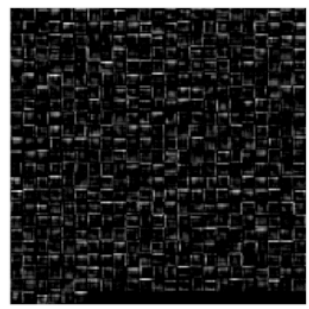

(vi)

(e) ClippedReLU of different depths in the network layer

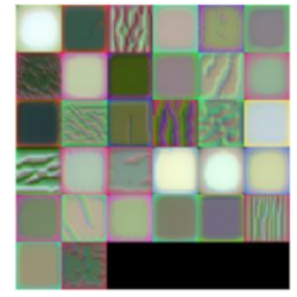

(i)

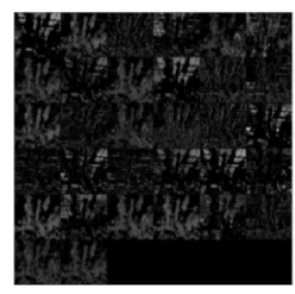

(ii)

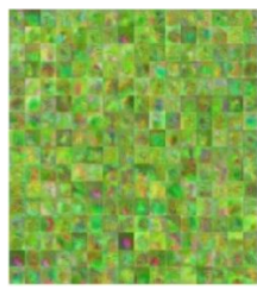

(iii)

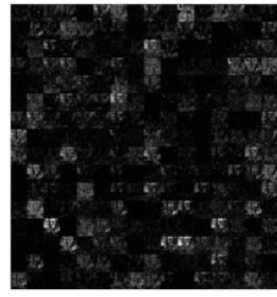

(iv)

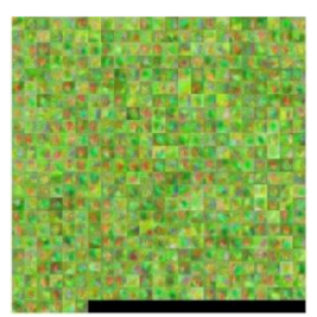

(v)

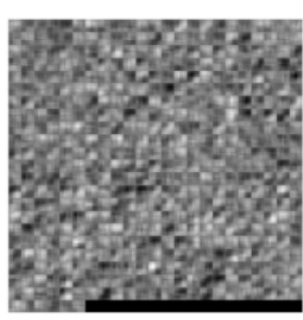

(vi)

(f) Average pooling of different depths in the network layer

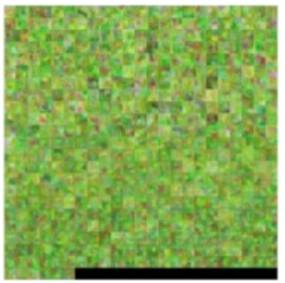

(i)

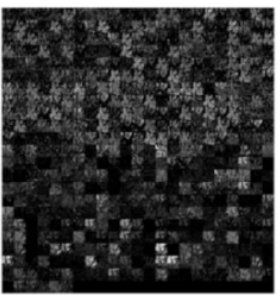

(ii)

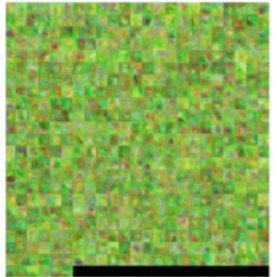

(iii)

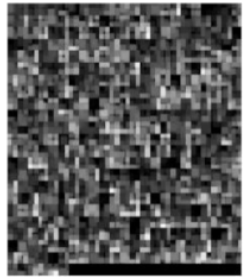

(iv)

Figure 10. Cont. 


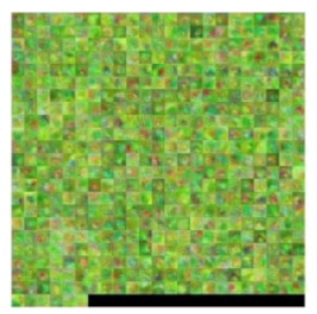

(v)

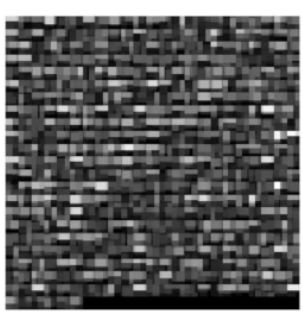

(vi)

(g) Max pooling of different depths in the network layer

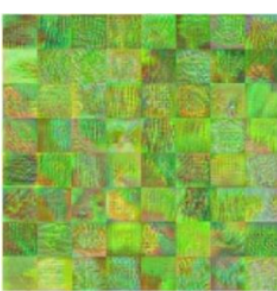

(i)

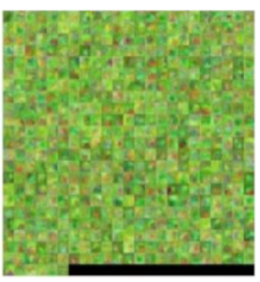

(v)

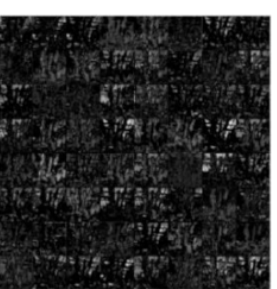

(ii)

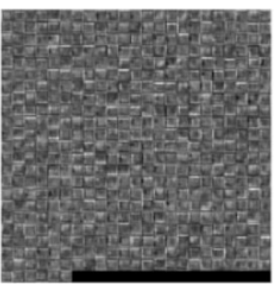

(vi)

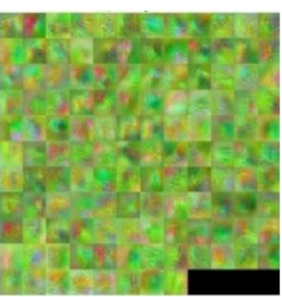

(iii)

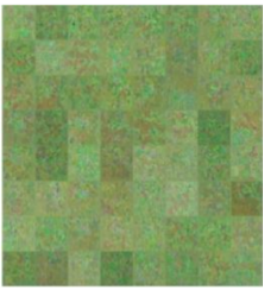

(vii)

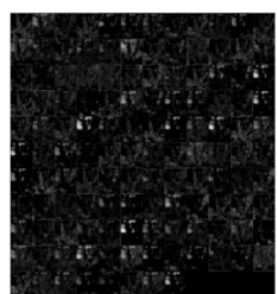

(iv)

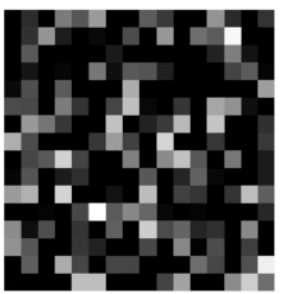

(viii)

(h) Addition of different depths in the network layer

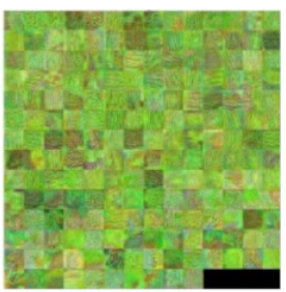

(i)

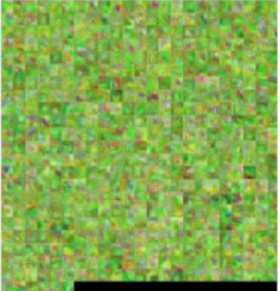

(v)

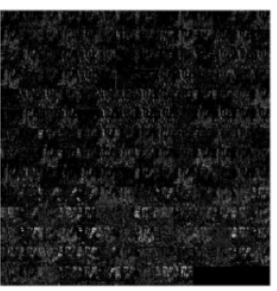

(ii)

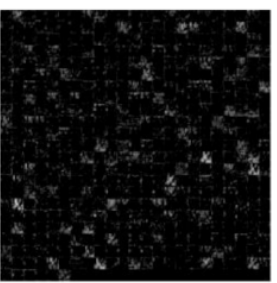

(vi)

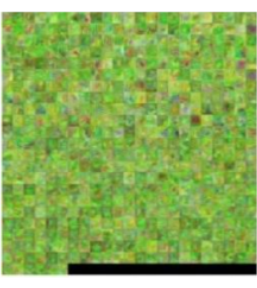

(iii)

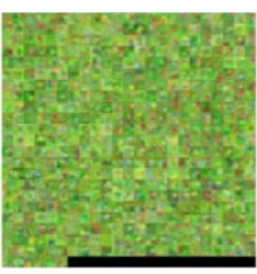

(vii)

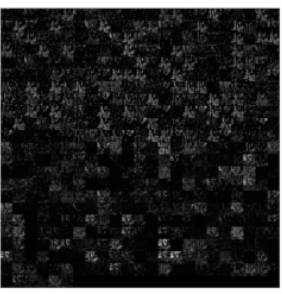

(iv)

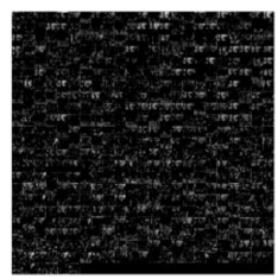

(viii)

(i) Depth concatenation of different depths in the network layer

Figure 10. Cont. 


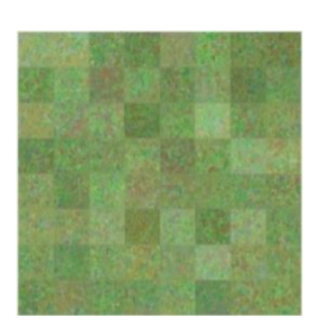

(i)

(j) Concatenation layer

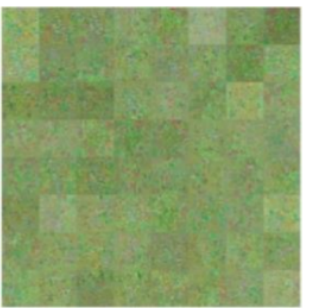

(i)

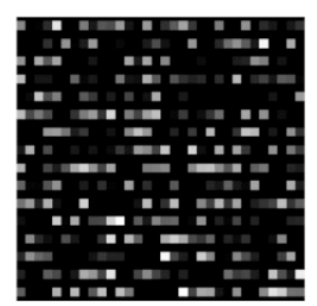

(ii)

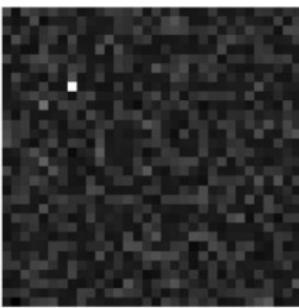

(ii)

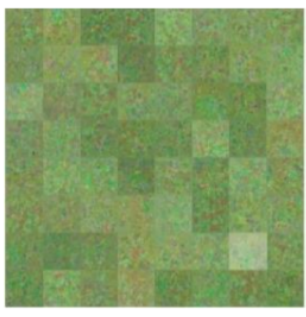

(iii)

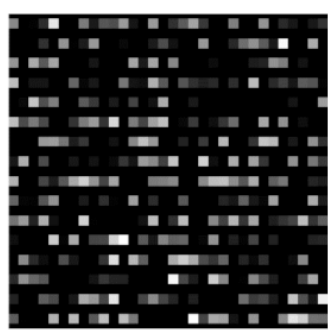

(iv)

(k) Dropout layer

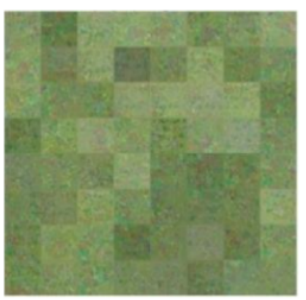

(iii)

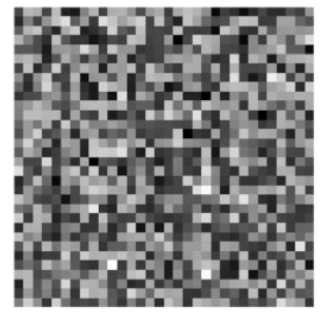

(iv)

(1) Group normalization of different depths in the network layer

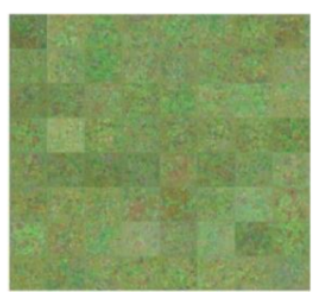

(i)

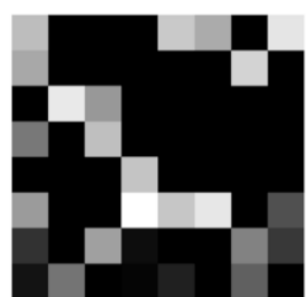

(ii)

(m) Global average pooling in the network layer

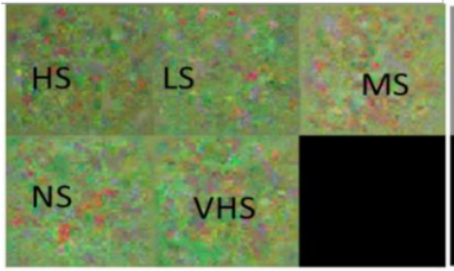

(i)

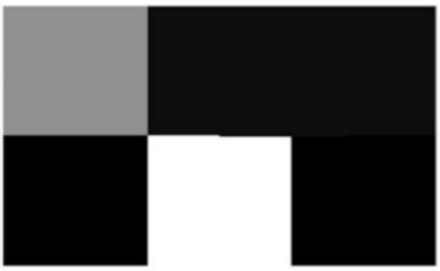

(ii)

(n) Fully connected network layer

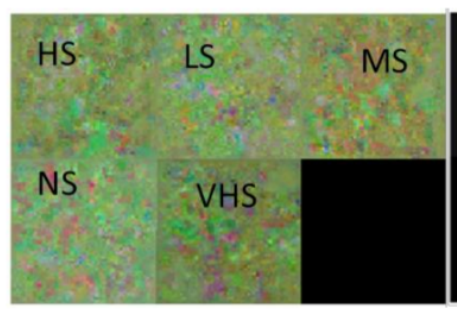

(i)

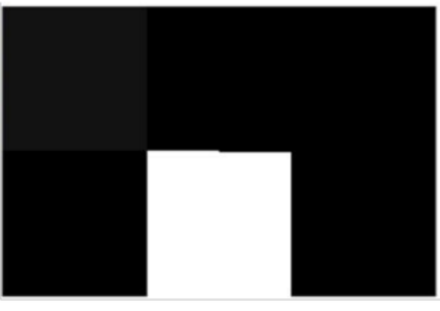

(ii)

(o) Softmax in the network layer

Figure 10. Visualization of network features and layer activations at different depths of the network. 


\subsubsection{Convolution Layer}

In the shallow depth of the network (layer 2), the input image size is $240 \times 240$ with 32 filters (Figure 10a(i)), and the weight and bias for learnable parameters are $3 \times 3 \times 3 \times 32$ and $1 \times 1 \times 32$, respectively. In the middle depth (139th layer), the input image size is $15 \times 15$ with 256 filters (Figure 10a(iii)), and the weight and bias for learnable parameters are $3 \times 3 \times 128 \times 256$ and $1 \times 1 \times 256$, respectively. In the deep depth (layer 313), the image size is $8 \times 8$ with 512 filters (Figure $10 \mathrm{a}(\mathrm{v})$ ), and the weight and bias for learnable parameters are $3 \times 3 \times 512 \times 512$ and $1 \times 1 \times 512$, respectively. In the final convolution layer (411th), the image size is $1 \times 2$ with 64 filters (Figure 10a(vii)), and the weight and bias for learnable parameters are $3 \times 3 \times 128 \times 64$ and $1 \times 1 \times 64$, respectively. The learnable feature is the response of the $\mathrm{CNN}$ (convolution neural network) layer to an input, as shown in Figure 10a(ii,iv,vi,viii).

\subsubsection{Batch-Normalization Layer}

In the shallow depth layer (3rd), the input image size is $240 \times 240$ with 32 filters (Figure $10 \mathrm{~b}(\mathrm{i})$ ), and the offset and scale for learnable parameters are $1 \times 1 \times 32$ and $1 \times 1 \times 32$, respectively. In the middle depth layer (142th), the input image size is $15 \times 15$ with 256 filters (Figure 10b(iii)), and the offset and scale for learnable parameters are $1 \times 1 \times 256$ and $1 \times 1 \times 256$, respectively. In the deep depth (layer 316), the image size is $8 \times 8$ with 512 filters, and the offset and scale for learnable parameters are $1 \times 1 \times 512$ and $1 \times 1 \times 512$ (Figure $10 \mathrm{~b}(\mathrm{v})$ ), respectively. In the final convolution (layer 412), the image size is $1 \times 2$ with 64 filters (Figure $10 \mathrm{~b}$ (vii)), and the offset and scale for learnable parameters are $1 \times 1 \times 64$ and $1 \times 1 \times 64$, respectively. The learnable feature is the response of the batch-normalization layer, as shown in Figure $10 b(i i, i v, v i, v i i i)$.

\subsubsection{Rectified Linear Unit (ReLU) Layer}

In the shallow depth layer (4th), the input image size is $240 \times 240$ with 32 filters (Figure 10c(i)). In the middle depth (148th layer), the input image size is $8 \times 8$ with 512 filters (Figure 10c(iii)). In the deep depth layer (324th), the image size is $8 \times 8$ with 512 filters (Figure 10c(v)). In the final ReLU (layer 413), the image size is $1 \times 2$ with 64 filters (Figure 10c(vii)). The learnable feature is the response of the ReLU layer, as shown in Figure $10 \mathrm{c}(\mathrm{ii}, \mathrm{iv}, \mathrm{vi}, \mathrm{viii})$.

\subsubsection{LeakyReLU}

In the shallow depth (19th layer), the input image size is $240 \times 240$ with 32 filters (Figure 10d(i)). In the middle depth layer (221th layer), the input image size is $8 \times 8$ with 512 filters (Figure 10d(iii)). In the deep depth layer (327th), the image size is $8 \times 8$ with 512 filters (Figure 10d(v)). In the final LeakyReLU (layer 407th), the image size is $1 \times 2$ with 256 filters (Figure 10c(vii)). The learnable feature is the response of the LeakyReLU layer to an input, as shown in Figure 10d(ii,iv,vi,viii).

\subsubsection{ClippedReLU}

In the shallow depth (layer 26th), the input image size is $120 \times 120$ with 64 filters (Figure 10e(i)). In the middle depth layer (244th), the input image size is $8 \times 8$ with 512 filters (Figure 10d(iii)). In the final ClippedReLU (layer 339th), the image size is $8 \times 8$ with 512 filters (Figure 10d(v)). Figure 10e(ii,iv,vi) shows the learnable feature which is the response of a ClippedReLU layer to an input.

\subsubsection{Average Pooling}

In the shallow depth (layer 15th), the input image size is $240 \times 240$ with 32 filters (Figure 10f(i)). In the middle depth (120th layer), the input image size is $60 \times 60$ with 256 filters (Figure 10f(iii)). In the deep depth layer (160th), the image size is $8 \times 8$ with 512 filters (Figure $10 \mathrm{f}(\mathrm{v}))$. The learnable feature is the response of the average pooling layer to an input, as shown in Figure 10f(ii,iv,vi). 


\subsubsection{Max Pooling}

In the shallow depth (190th layer), the input image size is $60 \times 60$ with 512 filters (Figure $10 \mathrm{~g}(\mathrm{i})$ ). In the middle depth (252th layer), the input image size is $8 \times 8$ with 512 filters (Figure $10 \mathrm{~g}$ (iii)). In the deep layer (354th), the image size is $8 \times 8$ with 512 filters (Figure $10 \mathrm{~g}(\mathrm{v})$ ). The learnable feature which is the response of the maximum (max) pooling layer to an input termed "activation", as shown in Figure 10g(ii,iv,vi).

\subsubsection{Addition Layer}

In the shallow depth (55th layer), the input image size is $120 \times 120$ with 64 filters (Figure 10h(i)). In the middle depth layer (138th), the input image size is $60 \times 60$ with 128 filters (Figure 10h(iii)). In the deep depth layer (336th), the image size is $8 \times 8$ with 512 filters (Figure $10 \mathrm{~h}(\mathrm{v})$ ). In the final addition layer (402th), the image size is $1 \times 1$ with 256 filters (Figure 10h(vii)). The learnable feature is the response of the addition layer to an input, as shown in Figure 10h(ii,iv,vi,viii).

\subsubsection{Depth Concatenation}

In the shallow depth layer (56th), the input image size is $120 \times 120$ with 192 filters (Figure 10i(i)). In the middle depth (189th layer), the input image size is $60 \times 60$ with 512 filters (Figure 10i(iii)). In the deep depth layer (203th), the image size is $15 \times 15$ with 512 filters (Figure 10i(v)). In the final addition layer (307th), the image size is $15 \times 15$ with 512 filters (Figure 10i(vii)). The learnable feature, that is, the response of the depth concatenation layer to an input termed "activation", as shown in Figure 10i(ii,iv,vi,viii).

\subsubsection{Concatenation and Dropout}

In the deep layer, the image size of the concatenation layer (203th) is $1 \times 2$ with 256 filters (Figure 10j(i)). In the dropout layer (404th), the image size is $1 \times 2$ with 256 filters (Figure 10k(i)). This layer also removes $10 \%$ of the unused neurons from the network. The learnable feature is the response of the concatenation and dropout layers to an input, as shown in Figure 10j(ii) and 10k(ii), respectively.

\subsubsection{Group Normalization}

The image size of the group normalization layer (278th layer) is $1 \times 1$ with 1024 filters (Figure 101(i)). At layer 382th, the image size is $1 \times 1$ with 1024 filters (Figure 101(iii)). The learnable feature is the response of the group normalization layer to the input, as shown in Figure 101(ii,iv).

\subsubsection{Global Average Pooling}

We use global average pooling before the fully connected layer to reduce the network dimension. At the 414th layer, the image size is $1 \times 1$ with 64 filters (Figure $10 \mathrm{~m}(\mathrm{i})$ ). The learnable feature, that is, the response of the global average pooling layer to the input, is shown in Figure 10m(ii).

\subsubsection{Fully Connected Layer}

The fully connected layer (415th layer) connects all the neurons in the previous layer, then combines all the features learned by the previous layer to identify larger patterns to classify the images. The image size of this layer is $1 \times 1$ (Figure 10n(i)) with five stress conditions. The learnable features (indicated in black and gray) are the response of the fully connected layer to the input, as shown in Figure 10n(ii).

\subsubsection{Softmax Layer}

The softmax function normalizes the input in the channel dimension and is used for the probability distributions of the output with a scale ranging from 0 to 1 . The output of this layer is $1 \times 1$ (Figure $10 \mathrm{o}(\mathrm{i})$ ) with 5 outputs of the stress condition and their probability 
distribution. The learnable feature (indicated in black) is the response of the softmax layer to an input, is shown in Figure 10o(ii).

\section{Discussion}

We conducted a live demonstration in the orchard of the National Institute of Fruit and Tea Science in Tsukuba, Japan (June, 2021), and deployed the HortNet417v1 network using a laptop equipped with the BUFFALO wide-angle web camera BSW200MBK $(1920 \times 1080$ pixels: $30 \mathrm{fps})$ and Matlab R2020a. Twenty three plants were randomly selected, of which 19 were pot-cultivated and four were soil-grown in the open field. The network detects the water stress status of peach trees in real time with an accuracy rate of $73 \%$. However, under the same conditions, MobileNetv2 shows an accuracy of $75 \%$, which is slightly higher than the HortNet $417 \mathrm{v} 1$ network. We generated a t-SNE plot (Figures 11-13) to visualize the features of the first convolution, final convolution, and softmax activation layers and to evaluate the distance between various water-stress conditions of the pot-cultivated peach plants. The occlusion sensitivity in Figure 14 shows the change in the probability of belonging to the right stress condition, and also shows how the network learns features from the training data.
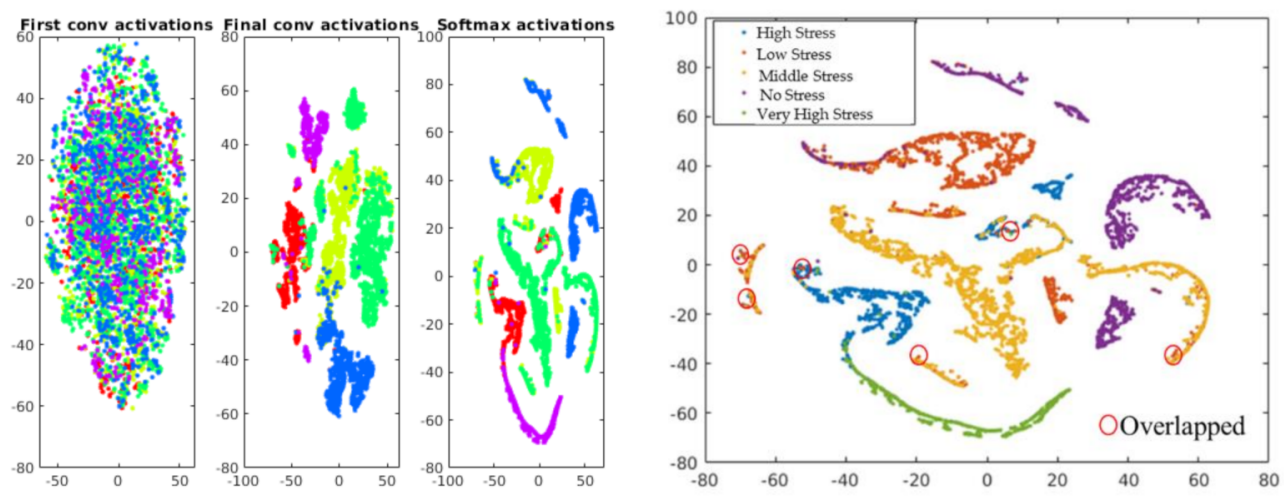

(a) Data distribution in different layers

(b) Classification in the output layer
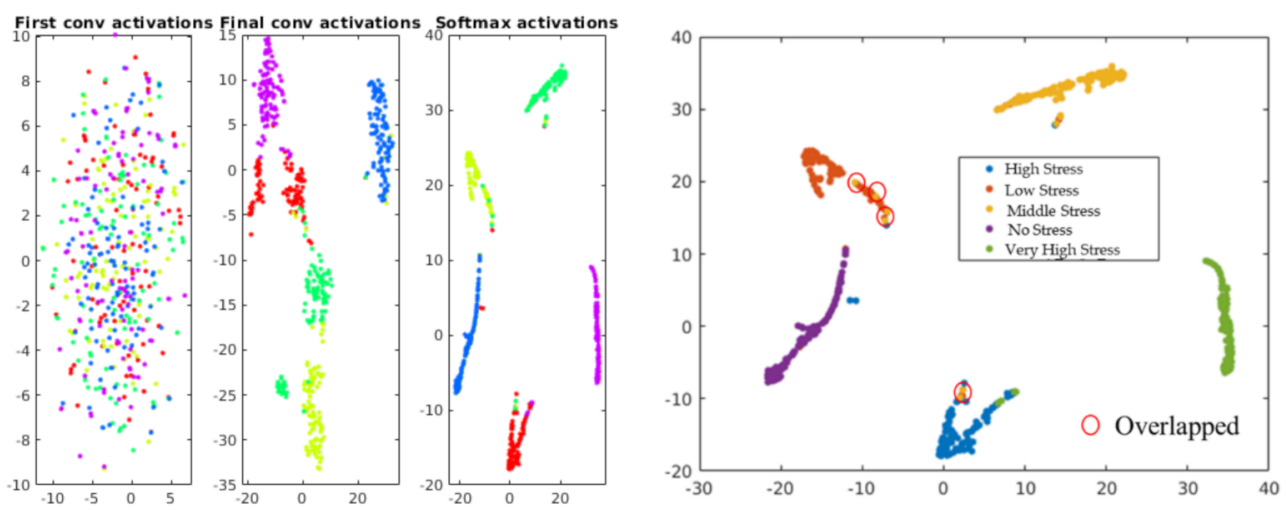

(c) Data distribution in different layers

(d) Classification in the output layer

Figure 11. Visualization of data distribution using t-SNE (t-distributed stochastic neighbor embedding) algorithm. 
Prediction mistake

Observation: 3

Actual: High Stress. Predicted: No Stress

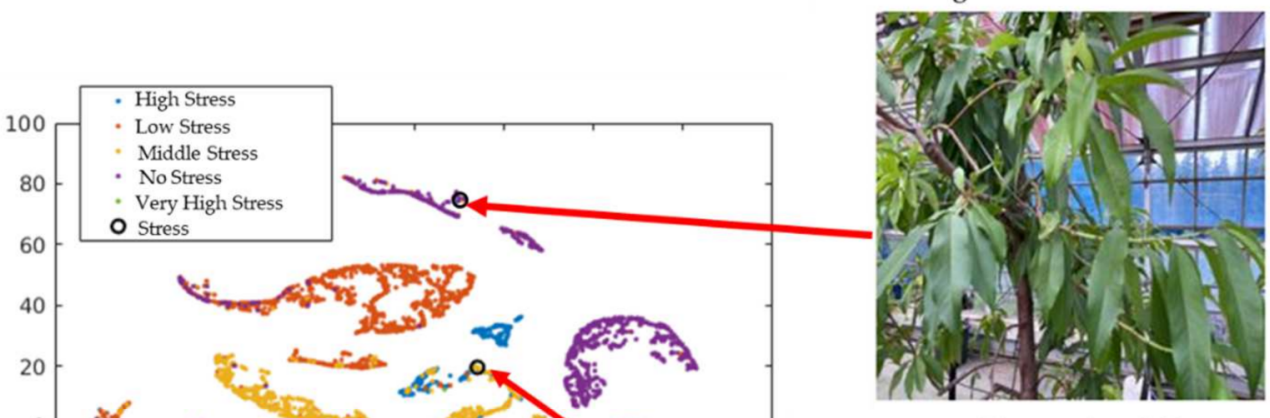

Observation: 346

Actual: High Stress. Predicted: High Stress

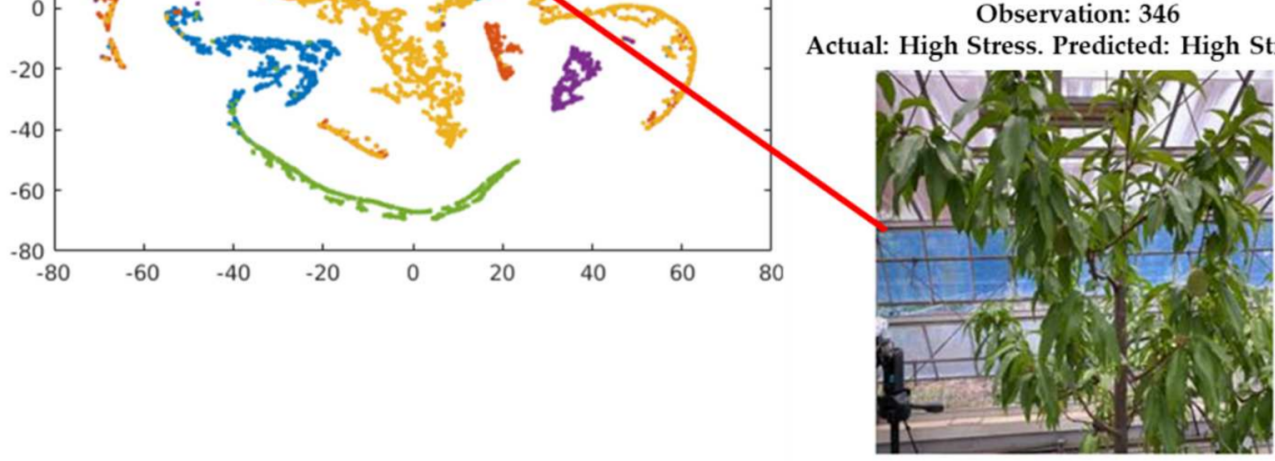

Prediction Accuracy

Figure 12. Exploration of observations in the t-SNE plot based on validation data.

Prediction mistake

Observation: 378

Actual: No Stress. Predicted: High Stress

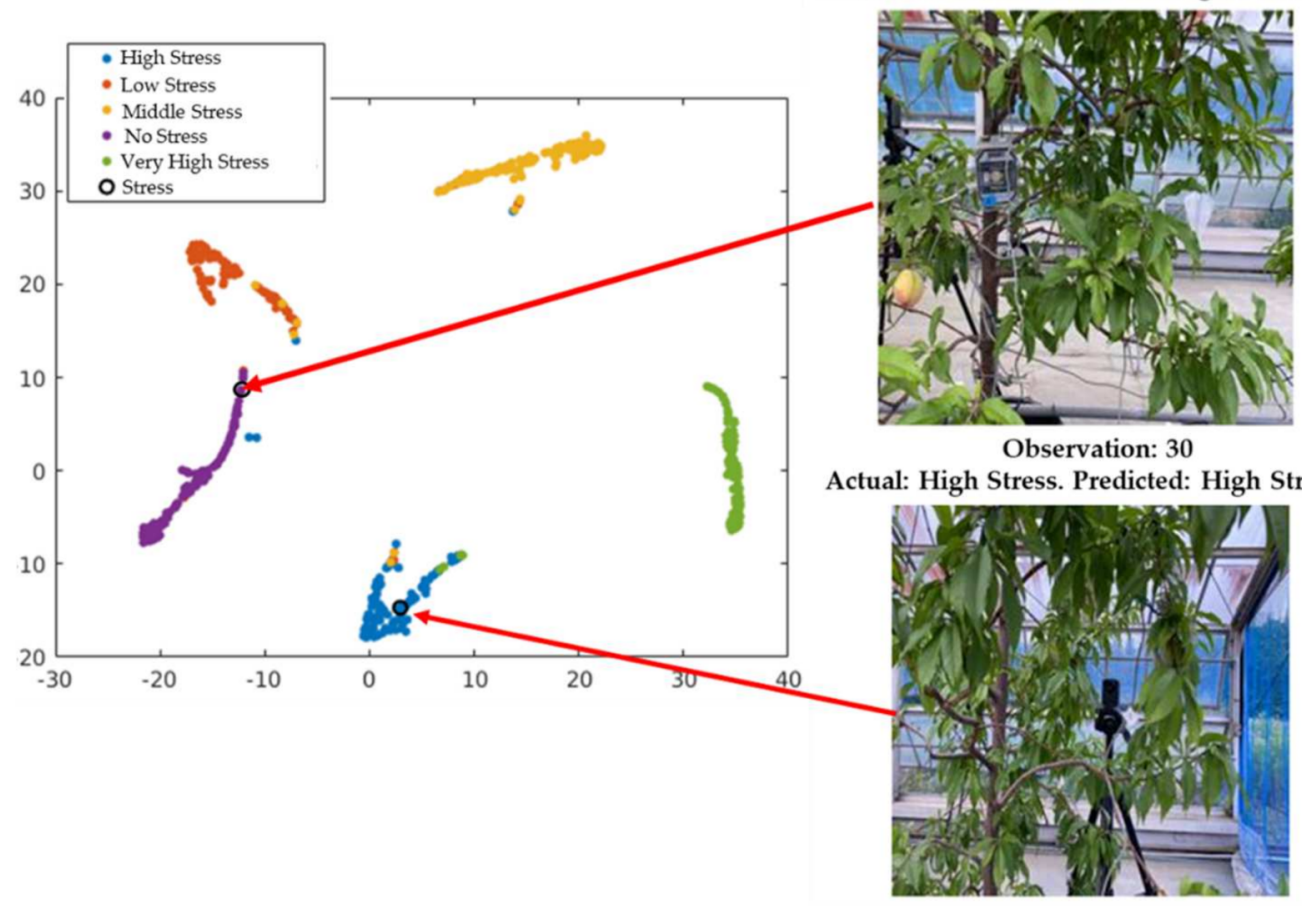

Prediction Accuracy

Figure 13. Exploration of observations in the t-SNE plot based on test data. 

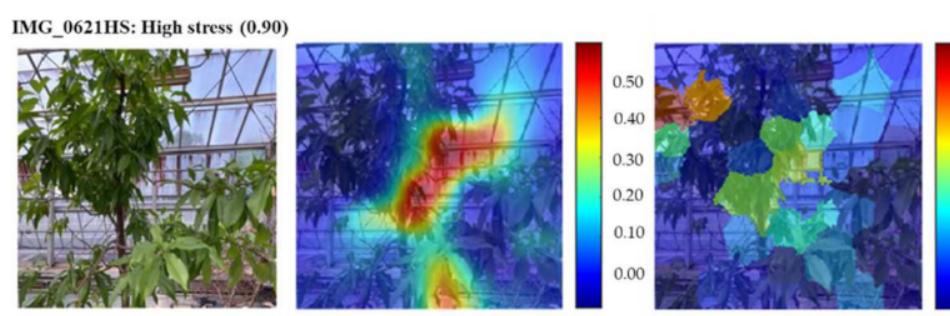

0.000020

IMG_5267HS: High stress (0.59)
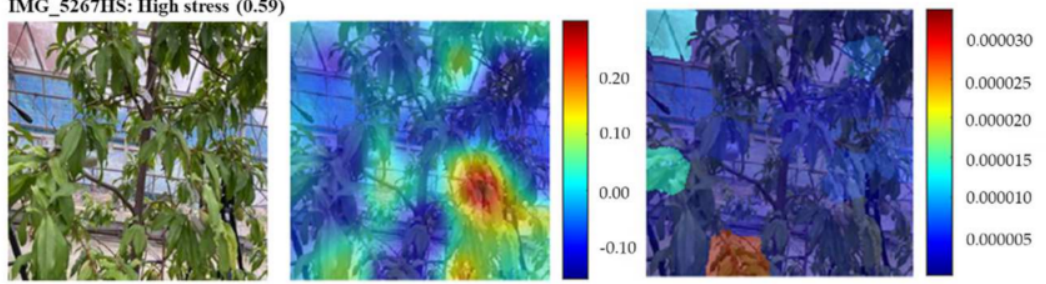

(i) Red, green and blue (RGB) Image (ii) Occlusion sensitivity (iii) LIME (locally interpretable model-agnostic explanation)

(a) High-stress conditions

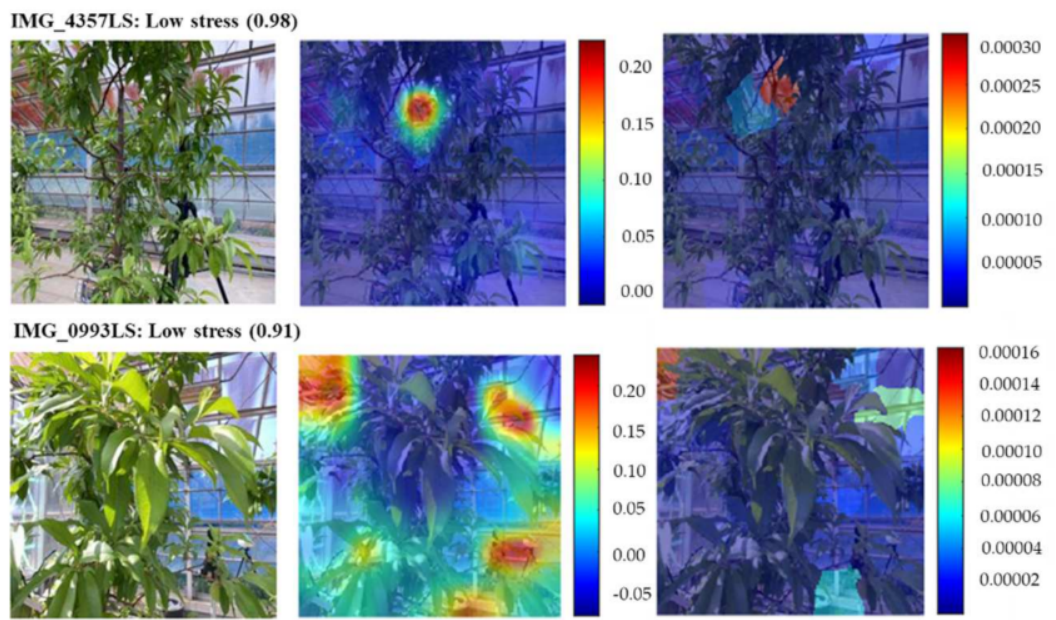

(i) RGB Image (ii) Occlusion sensitivity (iii) LIME

(b) Low-stress conditions

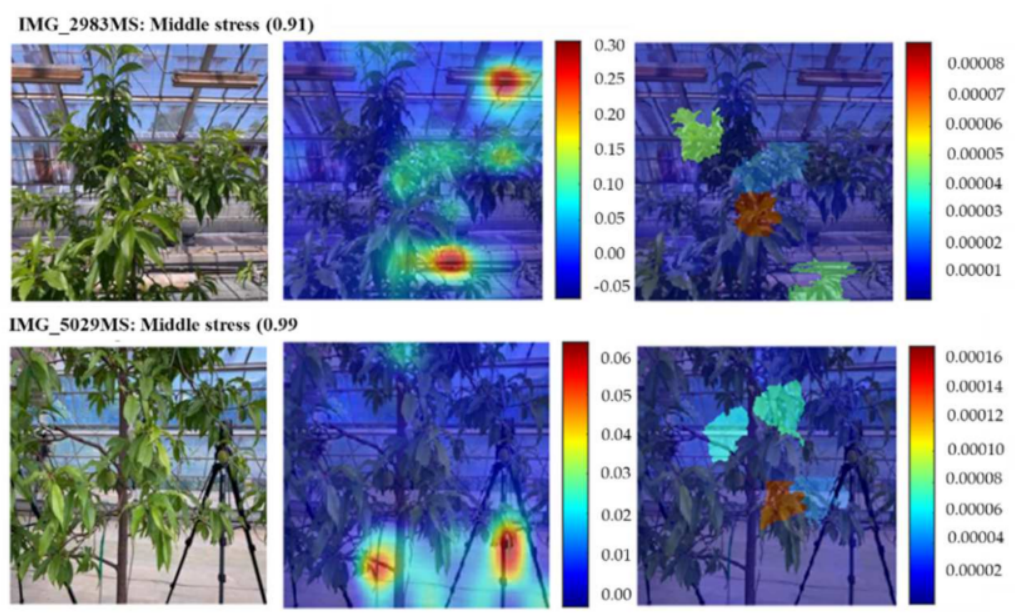

(i) RGB Image (ii) Occlusion sensitivity (iii) LIME

(c) Moderate-stress conditions

Figure 14. Cont. 

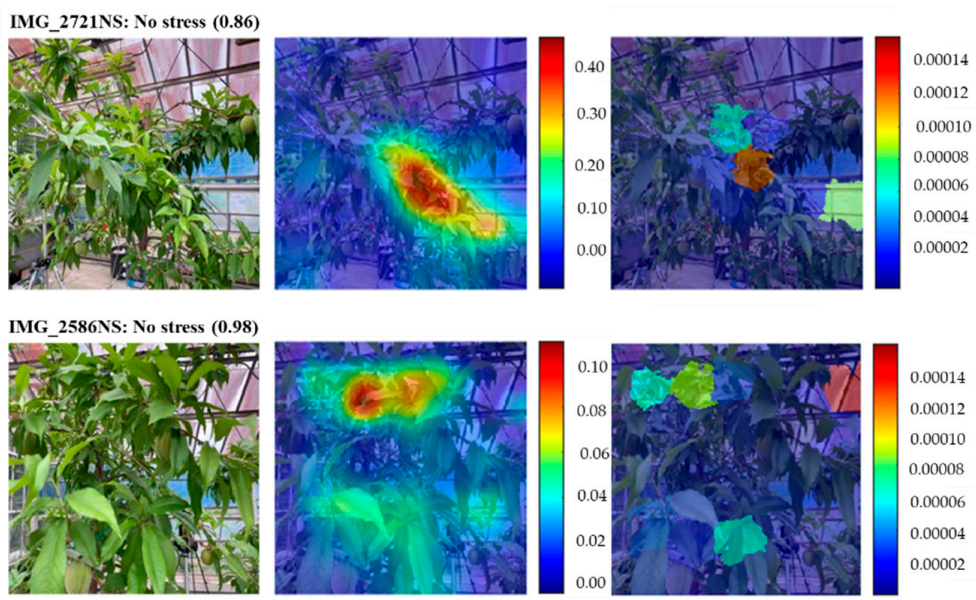

(i) RGB Image (ii) Occlusion sensitivity (iii) LIME

(d) No-stress conditions
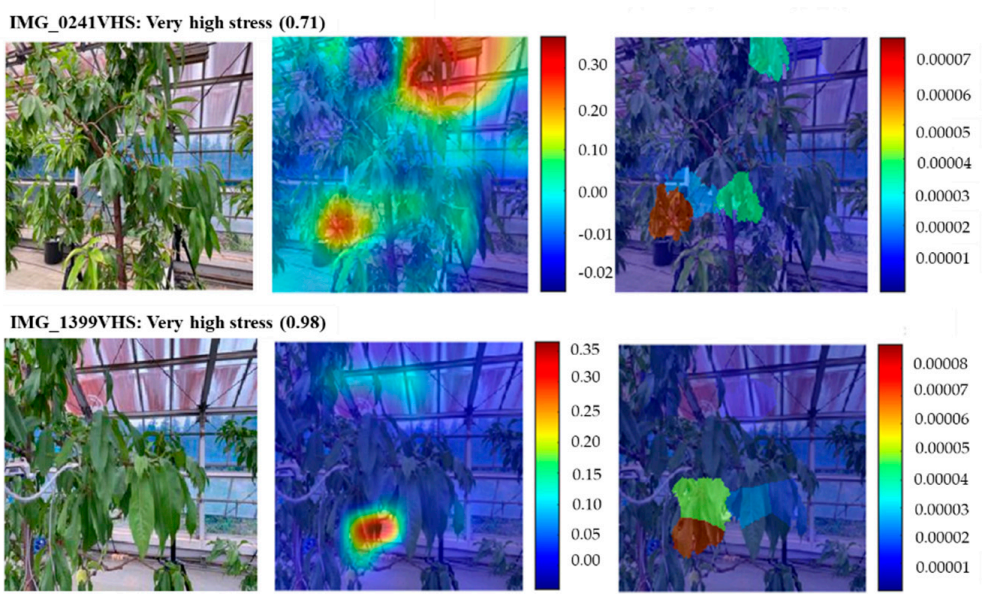

(i) RGB Image (ii) Occlusion sensitivity (iii) LIME

(e) Very-high-stress conditions

Figure 14. Visualization of the HortNet417v1 network decision behind the prediction of classification of the peach plant stress condition.

\subsection{Network Stress Condition Prediction Accuracy Evaluation Using the TSNE Algorithm}

Figure 11a visualizes the different intensities of the validation dataset distribution in the first convolution, final convolution, and softmax activation layers of the HortNet417v1 network. The majority of the stress conditions are separated, as shown in Figure 11b, while low stress data overlapped with moderate and high stress data. Figure 11c visualizes the different intensities of the test dataset distribution in the first convolution, final convolution, and softmax activation layers of the HortNet417v1 network. The majority of the stress conditions are separated, as shown in Figure 11d, while low stress data overlapped slightly with moderate and high stress data. This overlap is what we have observed in the validation and test data distribution and is caused by low, moderate, and high stress conditions. The images of these conditions are like to the images of the network and misclassified. By adding various patterns of the low, moderate, and high stress conditions, it is possible to solve this problem.

\subsection{Exploration of Observations in the t-SNE (t-Distributed Stochastic Neighbor Embedding) Plot}

The cross-validation classification in the last layer (output) is shown in Figure 12. The circle in the figure represents observation number 346, which provides the successful classification of the visualization of the cross-validation result of the 346th observation 
image from the validation data (actual and predicted stress conditions: high stress). This is difficult for the unexperienced human eye to detect. However, observer number 3 shows the misclassified visualization (actual: high stress; predicted: no stress) of the 3rd image from the validation dataset.

The classification ability of the output is shown in Figure 13. The circle in the figure shows observation number 30, which provides the successful classification (actual and predicted stress condition: high stress) of the visualization of the 30th image from the test data. This is also difficult for the unexperienced human eye to detect. However, observation number 378 shows the misclassified visualization of the 376th image from the test dataset (actual: high stress, predicted: no stress). The misclassification occurred due to the network's inability to learn the pattern differences among low, moderate, and high stress conditions.

\subsection{Predicted Result Evaluation Based on Occlusion Sensitivity and the LIME (Locally Interpretable Model-Agnostic Explanation) Technique}

The occlusion sensitivity of all images and the red color in LIME indicate the most important areas of the image that the network uses for classification decisions (Figure 14a (ii, iii; top and bottom). Figure 14a shows that the network predicted a high-stress condition with a probability of 0.90 and 0.59 , respectively. The occlusion sensitivity (Figure 14a(ii)) indicating the reason for the prediction is in the middle part for IMG_0621HS and the right corner of the lower part for IMG_5267HS. However, the LIME (Figure 14a(iii)) indicates that the reason for the prediction is in the left corner of the top part for IMG_0621HS and the middle to left corner of the lower part for IMG_5267HS.

In Figure 14b(i; top, bottom), the network predicted the low stress condition with a probability of 0.98 and 0.91 , respectively. The occlusion sensitivity (Figure $14 \mathrm{~b}(\mathrm{ii})$ ) indicating the reason for the prediction is shown in the middle of the top part (background) for IMG_4357LS. However, occlusion sensitivity indicates that the reason for the prediction is in the left and right corners of the top part and the right corner of the lower part for IMG_0993LS. The LIME (Figure 14b(iii)) suggests that the reason for the prediction is in the middle (mostly background) of the top part for IMG_0621HS and the left corner of the top part for MG_5267HS. Both the occlusion sensitivity and the LIME technique shows that the features of IMG_4357LS are learned incorrectly. This can be solved by adding more LS training data.

In Figure $14 \mathrm{c}(\mathrm{i}$; top, bottom), the predicted probability of the moderate stress condition is 0.91 and 0.99 , respectively. The occlusion sensitivity (Figure $14 \mathrm{c}(\mathrm{ii})$ ) indicates the reason for the prediction is in the right corner of the lower and top part, respectively, for IMG_2983MS. For image IMG_5029MS, the network also shows that the reason for the prediction is in the bottom of the lower part. In both cases, the right corner of the top part (background) for IMG_2983MS and the bottom of the lower part for IMG_5029MS indicate that the features are learned incorrectly. The LIME (Figure 14c(iii)) suggests that the reason for prediction is in the left, middle, and right parts for IMG_2983MS and the middle part for IMG_5029MS. In this case, the LIME technique indicates a more accurate position than the occlusion sensitivity.

In Figure 14d(i; top, bottom), the network predicts the no stress condition with a probability of 0.86 and 0.98 , respectively. The occlusion sensitivity (Figure $14 \mathrm{~d}(\mathrm{ii})$ ) indicates the reason for the prediction is the right corner of the lower part for IMG_2721NS and the middle of the top part for IMG_2586NS. LIME (Figure 14d(iii)) shows that the reason for the prediction is mainly in the middle and slightly right parts of IMG_2721NS. However, for image IMG_2856NS, the network shows the left and right corners of the top and middle of the lower part. In both IMG_2721NS and IMG_2856NS, the LIME technique also shows a slightly inaccurate position (lower and top right) as compared to the occlusion sensitivity.

In Figure 14e(i; top, bottom), the prediction probability for the very high stress condition is 0.71 and 0.98 , respectively. The occlusion sensitivity (Figure 14e(ii)) indicates the reason for the prediction is in the right corner of the top part for IMG_0241VHS and the middle to the left of the lower part for IMG_1399VHS. LIME (Figure 14e(iii)) shows that 
the main reason for the prediction is in the middle of the lower half of IMG_0241VHS and the left corner of the lower half of IMG_1399VHS.

\section{Conclusions}

This article mainly describes the HortNet417v1 architecture and provides network performance results by evaluating various performance indicators. We use the most descriptive approach to prove that HortNet417v1 can classify various water-stress conditions of the pot-cultivated peach plants. The main findings are as follows:

- Classification of uneven data sets under various stress conditions, which may lead to lack of information and diversity of images and stress conditions. Most pre-trained networks converge with higher accuracy after 25 epochs but HortNet $417 \mathrm{v} 1$ requires 36 epochs and more time to achieve higher accuracy. This response is because the weight of the pretrained model (Xception, ShuffleNet, and MobileNetv2) which is trained with millions of images, when actuated on a new training dataset, can converge at a faster rate than a network like HortNet417v1 in which network weights are randomly initialized instead of inherited from the previous model.

The research directions we are about to proceed along are as follows:

- In this experiment, we collect image data through a handheld mobile phone. In our next experiment, we will use some other fixed imaging platform surrounding the target plant to capture more time series data under various stress conditions and thus will improve the image data diversity and imbalance of the data amount between the stress conditions.

- Since the development of the network is a continuous process, the authors plan to modify the network structure, optimize the network hyperparameters, and train the network with more data to improve the prediction accuracy in real time. Then, this technology makes it possible to extend the study to a large agricultural area, not only for peach trees, but also for other types of fruit tree.

Author Contributions: Conceptualization, T.Y. and M.P.I.; methodology, M.P.I.; software, M.P.I.; validation, M.P.I. and T.Y.; formal analysis, M.P.I.; investigation, M.P.I.; resources, T.Y. and M.P.I.; data curation, T.Y. and M.P.I.; writing—original draft preparation, M.P.I.; writing-review and editing, M.P.I. and T.Y.; visualization, M.P.I.; supervision, T.Y.; project administration, T.Y.; funding acquisition, T.Y. All authors have read and agreed to the published version of the manuscript.

Funding: This work was supported by JSPS KAKENHI Grant Number JP21K05585.

Institutional Review Board Statement: Not applicable.

Informed Consent Statement: Not applicable.

Data Availability Statement: Data will be available upon completion of project and publication of the project report upon request to the corresponding author. Any request will be reviewed and approved by the sponsor, NARO, intellectual property department, researcher, and staff on the basis of the absence of competing interest. Once approved, data can be transferred after signing of a data access agreement and confidentiality agreement.

Conflicts of Interest: The authors declare that there are no conflicts of interest regarding the publication of this article.

\section{References}

1. Bryla, D.R.; Dickson, E.; Shenk, R.; Johnson, R.; Crisosto, C.H.; Trout, T.J. Influence of irrigation method and scheduling on patterns of soil and tree water status and its relation to yield and fruit quality in peach. J. Am. Soc. Hort. Sci. 2005, 40, 2118-2124.

2. Parker, M.L.; Hull, J.; Perry, R.L. Orchard floor management affects peach rooting. J. Am. Soc. Hort. Sci. 1993, 118, 714-718. [CrossRef]

3. Jones, H.G. Irrigation scheduling: Advantages and pitfalls of plant-based methods. J. Exp. Bot. 2004, 55, 2427-2436. [CrossRef] [PubMed]

4. Abrisqueta, I.; Conejero, W.; Valdes-Vela, M.; Vera, J.; Ortuño, M.F.; Ruiz-Sánchez, M.C. Stem water potential estimation of drip-irrigated early-maturing peach trees under Mediterranean conditions. Comput. Electron. Agric. 2015, 114, 7-13. [CrossRef] 
5. Mirás-Avalos, J.M.; Pérez-Sarmiento, F.; Alcobendas, R.; Alarcón, J.J.; Mounzer, O.; Nicolás, E. Using midday stem water potential for scheduling deficit irrigation in mid-late maturing peach trees under Mediterranean conditions. Irrig. Sci. 2016, $34,161-173$. [CrossRef]

6. LeCun, Y.; Bengio, Y.; Hinton, G. Deep learning. Nature 2015, 521, 436-444. [CrossRef]

7. Singh, A.K.; Ganapathysubramanian, B.; Sarkar, S.; Singh, A. Deep learning for plant stress phenotyping: Trends and future perspectives. Trends Plant Sci. 2018, 23, 883-898. [CrossRef]

8. Saleem, M.H.; Potgieter, J.; Arif, K.M. Plant disease detection and classification by deep learning. Plants $2019,8,468$.

9. Wakamori, K.; Mizuno, R.; Nakanishi, G.; Mineno, H. Multimodal neural network with clustering-based drop for estimating plant water stress. Comput. Electron. Agric. 2020, 168, 105118. [CrossRef]

10. Fricke, W. Water transport and energy. Plant Cell Environ. 2017, 40, 977-994. [CrossRef]

11. Kamarudin, M.H.; Ismail, Z.H.; Saidi, N.B. Deep learning sensor fusion in plant water stress assessment: A comprehensive review. Appl. Sci. 2021, 11, 1403. [CrossRef]

12. Chlingaryan, A.; Sukkarieh, S.; Whelan, B. Machine learning approaches for crop yield prediction and nitrogen status estimation in precision agriculture: A review. Comput. Electron. Agric. 2018, 151, 61-69. [CrossRef]

13. Simonyan, K.; Zisserman, A. A very deep convolutional networks for large-scale image recognition. In Proceeding of the 3rd International Conference on Learning Representations, San Diego, CA, USA, 7-9 May 2015.

14. He, K.; Zhang, X.; Ren, S.; Jian, S. Deep residual learning for image recognition. In Proceedings of the IEEE Conference on Computer Vision and Pattern Recognition, Las Vegas, NV, USA, 27-30 June 2016.

15. McCutchan, H.; Shackel, K.A. Stem-water potential as a sensitive indicator of water stress in prune trees (Prunus domestica L. cv. French). J. Am. Soc. Hort. Sci. 1992, 117, 607-611.

16. Shackel, K.A.; Ahmadi, H.; Biasi, W.; Buchner, R.; Goldhamer, D.; Gurusinghe, S.; Hasey, J.; Kester, D.; Krueger, B.; Lampinen, B.; et al. Plant water status as an index of irrigation need in deciduous fruit trees. HortTechnology. 1997, 7, $23-29$.

17. Zhang, Q.; Liu, Y.; Gong, C.; Chen, Y.; Yu, H. Application of deep learning for dense scenes analysis in agriculture: A review. Sensors 2020, 20, 1520. [CrossRef]

18. Nair, V.; Geoffrey, E.H. Rectified linear units improve restricted Boltzmann machines. In Proceedings of the 27th International Conference on Machine Learning, Haifa, Israel, 21-24 June 2010.

19. Hannan, A.; Case, C.; Casper, J.; Catanzaro, B.; Diamos, G.; Elsen, E.; Prenger, R.; Satheesh, S.; Sengupta, S.; Coates, A.; et al. Deep Speech: Scaling up end-to-end speech recognition. arXiv 2014, arXiv:1412.5567, 12.

20. Ioffe, S.; Szegedy, C. Batch normalization: Accelerating deep network training by reducing internal covariate shift. arXiv 2015, 37, 11.

21. Wu, Y.; He, K. Group Normalization. arXiv 2018, arXiv:1803.0849, 10.

22. Scherer, D.; Mueller, A.; Behnke, S. Evaluation of pooling operations in convolution architectures for object recognition. In Proceedings of the 20th International Conference on Artificial Neural Networks, Thessaloniki, Greece, 15-18 September 2010.

23. Nagi, J.; Ducatelle, F.; Di Caro, G.A.; Ciresan, D.; Meier, U.; Giusti, A.; Nagi, F.; Schmidhuber, J.; Gambardella, L.M. Max-pooling convolutional neural networks for vision-based hand gesture recognition. In Proceedings of the IEEE International Conference on Signal and Image Processing Applications, Kuala Lumpur, Malaysia, 16-18 November 2011.

24. Yani, M.; Irawan, B.; Setiningsih, C. Application of transfer learning using convolutional neural network method for early detection of Terry's nail. J. Phys. Conf. Ser. 2019, 1201, 10. [CrossRef]

25. Krizhevsky, A.; Sutskever, I.; Hinton, G.E. ImageNet classification with deep convolution neural networks. Adv. Neural Inf. Proc. Syst. 2012, 25, 1097-1105.

26. Zoph, B.; Vasudevan, V.; Shlens, J.; Le, Q.V. Learning transferable architectures for scalable image recognition. arXiv 2017, arXiv:1707.07012, 14.

27. He, K.; Zhang, X.; Ren, S.; Jian, S. Identity mappings in deep residual networks. In Proceedings of the 14th European Conference on Computer Vision, Amsterdam, The Netherlands, 11-14 October 2016.

28. Chollet, F. Xception: Deep learning with depthwise separable convolutions. arXiv 2017, arXiv:1610.02357, 8.

29. Zhang, X.; Xinyu, Z.; Mengxiao, L.; Jian, S. ShuffleNet: An extremely efficient convolutional neural network for mobile devices. arXiv 2017, arXiv:1707.01083, 9.

30. Landola, F.N.; Song, H.; Matthew, W.; Moskewicz, K.A.; William, J.D.; Kurt, K. SqueezeNet: AlexNet-level accuracy with 50x fewer parameters and $<0.5 \mathrm{MB}$ model size. In Proceedings of the International Conference on Learning Representations, Toulon, France, 24-26 April 2016.

31. Szegedy, C.; Liu, W.; Jia, Y.; Sermanet, P.; Reed, S.; Anguelov, D.; Erhan, D.; Vanhoucke, V.; Rabinovich, A. Going deeper with convolutions. arXiv 2014, arXiv:1409.4842, 12.

32. Sandler, M.; Howard, A.; Zhu, M.; Zhmoginov, A.; Chen, L.C. MobileNetV2: Inverted residuals and linear bottlenecks. In Proceedings of the IEEE/CVM Conference on Computer Vision and Pattern Recognition, Salt Lake City, UT, USA, 23-28 June 2018.

33. Van der Maaten, L.; Geoffrey, H. Visualizing data using t-SNE. J. Mac. Learn. Res. 2008, 9, 2579-2605.

34. Zeiler, M.D.; Fergus, R. Visualizing and understanding convolutional networks. In Computer Vision-ECCV 2014; Fleet, D., Pajdla, T., Schiele, B., Tuytelaars, T., Eds.; Springer: Cham, Switzerland, 2014. 
35. Ribeiro, M.T.; Singh, S.; Guestrin, C. "Why should I trust you?": Explaining the predictions of any classifier. In Proceedings of the 22nd ACM SIGKDD International Conference on Knowledge Discovery and Data Mining, San Francisco, CA, USA, 26-29 August 2016.

36. Goodfellow, I.; Bengio, Y.; Courville, A. Deep Learning; MIT Press: Cambridge, UK, 2016. 